\title{
Homoclinic orbits, and self-excited and hidden attractors in a Lorenz-like system describing convective fluid motion
}

\section{Homoclinic orbits, and self-excited and hidden attractors}

\author{
G.A. Leonov ${ }^{1}$, N.V. Kuznetsov ${ }^{1,2, a}$, and T.N. Mokaev ${ }^{1,2}$ \\ 1 Faculty of Mathematics and Mechanics, St. Petersburg State University, St. Petersburg, \\ Russia \\ 2 Department of Mathematical Information Technology, University of Jyväskylä, \\ Jyväskylä, Finland
}

Received 17 March 2015 / Received in final form 20 May 2015

Published online 27 July 2015

\begin{abstract}
In this paper, we discuss self-excited and hidden attractors for systems of differential equations. We considered the example of a Lorenz-like system derived from the well-known GlukhovskyDolghansky and Rabinovich systems, to demonstrate the analysis of self-excited and hidden attractors and their characteristics. We applied the fishing principle to demonstrate the existence of a homoclinic orbit, proved the dissipativity and completeness of the system, and found absorbing and positively invariant sets. We have shown that this system has a self-excited attractor and a hidden attractor for certain parameters. The upper estimates of the Lyapunov dimension of self-excited and hidden attractors were obtained analytically.
\end{abstract}

\section{Introduction: Self-excited and hidden attractors}

When the theories of dynamical systems and oscillations were first developed (see, e.g., the fundamental works of Poincare and Lyapunov), researchers mainly focused on analyzing equilibria stability and the birth of periodic oscillations. The structures of many applied systems (see, e.g., the Rayleigh [148], Duffing [41], van der Pol [144], Tricomi [162], and Beluosov-Zhabotinsky [13] systems) are such that it is almost obvious that periodic oscillations exist, because the oscillations are excited by an unstable equilibrium. This meant that scientists of that time could compute such oscillations (called self-excited oscillations) by constructing a solution using initial data from a small neighborhood of the equilibrium, observing how it is attracted, and visualizing the oscillation (standard computational procedure). In this procedure, computational methods and the engineering notion of a transient process were combined to study oscillations.

\footnotetext{
a e-mail: nkuznetsov239@gmail.com
} 


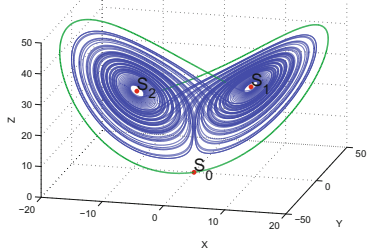

(a) Initial data near the equilibrium $S_{0}$

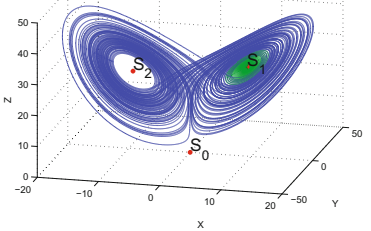

(b) Initial data near the equilibrium $S_{1}$

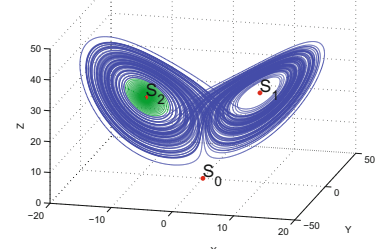

(c) Initial data near the equilibrium $S_{2}$

Fig. 1. Numerical visualization of the classical, self-excited, chaotic attractor in the Lorenz system $\dot{x}=10(y-x), \dot{y}=28 x-y-x z, \dot{z}=-8 / 3 z+x y$ by the trajectories that start in small neighborhoods of the unstable equilibria $S_{0,1,2}$. Here the separation of the trajectory into transition process (green) and approximation of attractor (blue) is rough.

At the end of the 19th century Poincare considered Newtonian dynamics of the three body problem, and revealed the possibility of more complicated behaviors of orbits "so tangled that I cannot even begin to draw them". He arrived at the conclusion that "it may happen that small differences in the initial positions may lead to enormous differences in the final phenomena". Further analyses and visualizations of such complicated "chaotic" systems became possible in the middle of the 20th century after the appearance of powerful computational tools.

An oscillation can generally be easily numerically localized if the initial data from its open neighborhood in the phase space (with the exception of a minor set of points) lead to a long-term behavior that approaches the oscillation. From a computational perspective, such an oscillation (or set of oscillations) is called an attractor, and its attracting set is called the basin of attraction (i.e., a set of initial data for which the trajectories tend to the attractor).

The first well-known example of a visualization of chaotic behavior in a dynamical system is from the work of Lorenz [122]. It corresponds to the excitation of chaotic oscillations from unstable equilibria, and could have been found using the standard computational procedure (see Fig. 1). Later, various self-excited chaotic attractors were discovered in many continuous and discrete systems (see, e.g., $[23,26,31,50,123$, $150,156])$.

The study of an autonomous (unperturbed) system typically begins with an analysis of the equilibria, which are easily found numerically or analytically. Therefore, from a computational perspective, it is natural to suggest the following classification of attractors $[80,108,111,112]$, which is based on the simplicity of finding their basins of attraction in the phase space:

Definition 1. [80,108,111,112] An attractor is called a self-excited attractor if its basin of attraction intersects with any open neighborhood of a stationary state (an equilibrium), otherwise it is called a hidden attractor.

The basin of attraction for a hidden attractor is not connected with any equilibrium. For example, hidden attractors are attractors in systems with no equilibria or with only one stable equilibrium (a special case of the multistability: coexistence of attractors in multistable systems). Note that multistability can be inconvenient in various practical applications (see, for example, discussions on problems related to the synchronization of coupled multistable systems in $[60,61,70,142])$. Coexisting self-excited attractors can be found using the standard computational procedure ${ }^{1}$,

\footnotetext{
${ }^{1}$ We have not discussed possible computational difficulties such as Wada and riddled basins.
} 


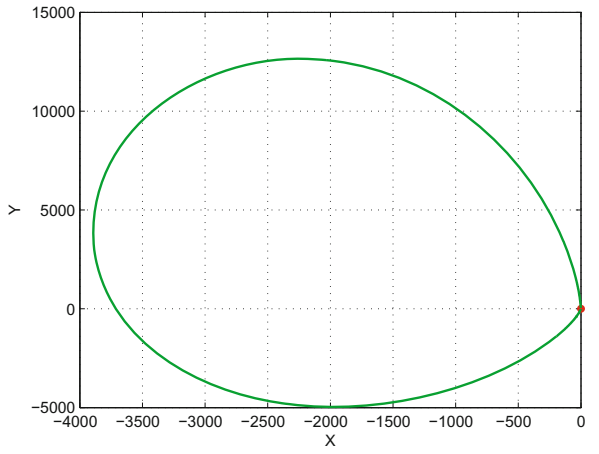

(a)

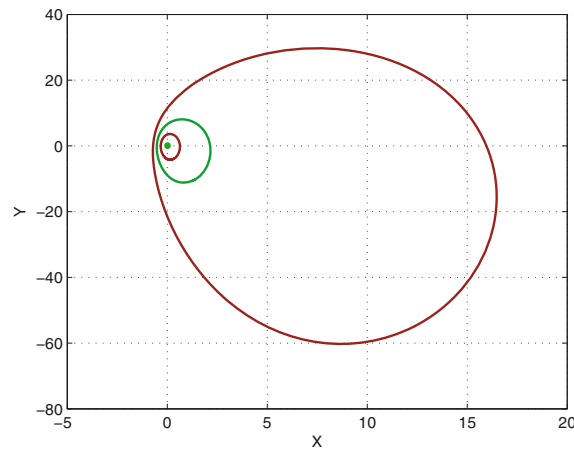

(b)

Fig. 2. Visualization of four limit cycles (green represents stable and red represents unstable) in a two-dimensional polynomial quadratic system $\dot{x}=-\left(a_{1} x^{2}+b_{1} x y+c_{1} y^{2}+\alpha_{1} x+\beta_{1} y\right), \dot{y}=$ $-\left(a_{2} x^{2}+b_{2} x y+c_{2} y^{2}+\alpha_{2} x+\beta_{2} y\right)$, for the coefficients $a_{1}=b_{1}=\beta_{1}=-1, c_{1}=\alpha_{1}=0$, $b_{2}=-2.2$, and $c_{2}=-0.7, a_{2}=10, \alpha_{2}=72.7778$, and $\beta_{2}=-0.0015$. Localization of three nested limit cycles around the stable zero point (green dot) and one limit cycle to the left of the straight line $x=-1$.

whereas there is no standard way of predicting the existence or coexistence of hidden attractors in a system.

Hidden attractors arise in connection with various fundamental problems and applied models. The problem of analyzing hidden periodic oscillations first arose in the second part of Hilbert's 16th problem (1900), which considered the number and mutual disposition of limit cycles in two-dimensional polynomial systems [51]. The first nontrivial results were obtained by Bautin (see, e.g., [12]), which were devoted to the theoretical construction of three nested limit cycles around one equilibrium in quadratic systems. Bautin's method can only be used to construct nested, smallamplitude limit cycles, which can hardly be visualized. However, recently an analytical approach has been developed, which can be used to effectively visualize nested, normal amplitude limit cycles in quadratic systems [75, 108,113].

Later, in the 1950s-1960s, studies of the well-known Markus-Yamabe's [125], Aizerman's [2], and Kalman's [59] conjectures on absolute stability led to the discovery of the possible coexistence of a hidden periodic oscillation and a unique stable stationary point in automatic control systems (see [10,15,20,45,79,104,105, 143]; the corresponding discrete examples were considered in [4]).

The Rabinovich system [146] and the Glukhovsky-Dolghansky system [48] are among the first known chaotic systems that have hidden chaotic attractors [72,91]. The first one describes the interaction of plasma waves and was considered in 1978 by Rabinovich [140,146] Another is a model of convective fluid motion and was considered in 1980 by Glukhovsky and Dolghansky [48] (which we consider in the remainder of this paper).

Hidden oscillations appear naturally in systems without equilibria, describing various mechanical and electromechanical models with rotation, and electrical circuits with cylindrical phase space. One of the first examples is from a 1902 paper [154] in which Zommerfield analyzed the vibrations caused by a motor driving an unbalanced weight and discovered the so-called Zommerfield effect (see, e.g., [18,42]). Another well-known chaotic system without equilibria is the Nosè-Hoover oscillator $[53,130]$ (see also the corresponding Sprott system, which was discovered independently $[156,157])$. In 2001, a hidden chaotic attractor was reported in a power system with no equilibria [165] (and references within). 
After the idea of a "hidden attractor" was introduced and the first hidden Chua attractor was discovered [69,73,76,80,103,111,112], hidden attractors have received much attention. Results on the study of hidden attractors were presented in a number of invited survey and plenary lectures at various international conferences ${ }^{2}$. In 2012, an invited comprehensive survey on hidden attractors was prepared for the International Journal of Bifurcation and Chaos [108].

Many researchers are currently studying hidden attractors. Hidden periodic oscillations and hidden chaotic attractors have been studied in models such as phaselocked loops [68,71], Costas loops [16], drilling systems [65,110], DC-DC converters [176], aircraft control systems [5], launcher stabilization systems [6], plasma waves interaction [72], convective fluid motion [91], and many others models (see, e.g., $[21,24,27,28,38,57,58,63,67,84,118-120,129,134-139,152,158,166-170,175])$.

Similar to autonomous systems, when analyzing and visualizing chaotic behaviors of nonautonomous systems, we can consider the extended phase space and introduce various notions of attractors (see, e.g., $[25,66]$ ). Alternatively, we can regard time $t$ as a phase space variable that obeys the equation $\dot{t}=1$. For systems that are periodic in time, we can also introduce a cylindrical phase space and consider the behavior of trajectories on a Poincare section.

The consideration of system equilibria and the notions of self-excited and hidden attractors are natural for autonomous systems, because their equilibria can be easily found analytically or numerically. However, we may use other objects to construct transient processes that lead to the discovery of chaotic sets. These objects can be constructed for the considered system or its modifications (i.e., instead of analyzing the scenario of the system transiting into chaos, we can synthesis a new transition scenario). For example, we can use perpetual points [145] or the equilibria of the complexified system [134]. A periodic solution or homoclinic trajectory can be used in a similar way (some examples of theoretical studies can be found in $[22,117,127,153]$; however the presence of chaotic behavior in the considered examples may not imply the existence of a chaotic attractor, which can be numerically visualized using the standard computational procedure).

For nonautonomous systems, depending on the physical problem statement, the notion of self-excited and hidden attractors can be introduced with respect to either the stationary states $\left(x(t) \equiv x_{0} \forall t\right)$ of the considered nonautonomous system, the stationary points of the system at fixed initial time $t=t_{0}$, or the corresponding system without time-varying excitations. If the discrete dynamics of the system are considered on a Poincare section, then we can also use stationary or periodic points on the section that corresponds to a periodic orbit of the system (the consideration of periodic orbits is also natural for discrete systems).

In the following, we consider an example of a nonautonomous system (a forced Duffing oscillator), so that we can visualize the chaotic behavior. The classical example of a self-excited chaotic attractor (Fig. 3) in a Duffing system $\ddot{x}+0.05 \dot{x}+x^{3}=7.5 \cos (t)$ was numerically constructed by Ueda in 1961, but it become well-known much later [163]. To construct a self-excited chaotic attractor in this system, we use a transient process from the zero equilibrium of the unperturbed autonomous system (i.e., without $\cos (t))$ to the attractor (Fig. 3) in the forced system.

\footnotetext{
${ }^{2}$ X Int. Workshop on Stability and Oscillations of Nonlinear Control Systems (Russia, 2008), Physics and Control [103] (Italy, 2009), 3rd International Conference on Dynamics, Vibration and Control (Hangzhou, China, 2010), IFAC 18th World Congress [105] (Italy, 2011), IEEE 5th Int. Workshop on Chaos-Fractals Theories and Applications [106] (Dalian, China, 2012), International Conference on Dynamical Systems and Applications (Ukraine, 2012), Nostradamus (Czech Republic, 2013) [107] and others.
} 


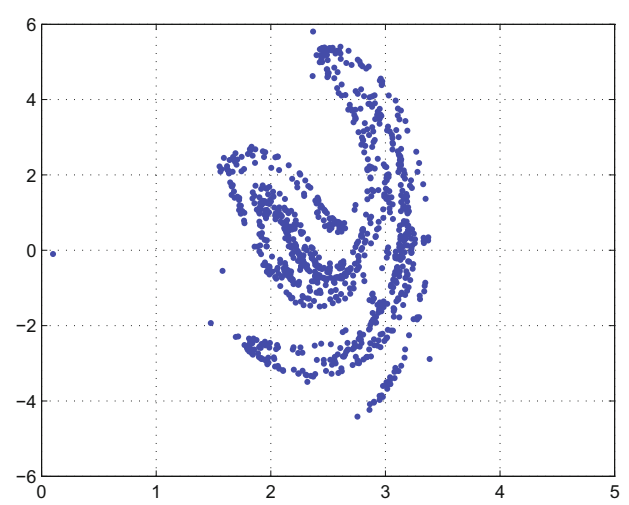

Fig. 3. Forced Duffing oscillator: $\ddot{x}+0.05 \dot{x}+x^{3}=7.5 \cos (t)$. The $(x, \dot{x})$ plane is mapped into itself by following the trajectory for time $0 \leq t \leq 2 \pi$. After a transition process a trajectory from the vicinity of the zero stationary point of the unperturbed Duffing oscillator (without $7.5 \cos (t))$ visualizes a self-excited chaotic attractor in the forced oscillator.

Note that if the attracting domain is the whole state space, then the attractor can be visualized by any trajectory and the only difference between computations is the timing of the transient process.

\section{A Lorenz-like system}

Consider a three-dimensional Lorenz-like system

$$
\left\{\begin{array}{c}
\dot{x}=-\sigma(x-y)-a y z \\
\dot{y}=r x-y-x z \\
\dot{z}=-b z+x y
\end{array}\right.
$$

For $a=0$, system (1) coincides with the classical Lorenz system [122]. For $\sigma>$ ar and $b=1$ after a linear change of variables [99]

$$
x \rightarrow x, \quad y \rightarrow \frac{C}{\sigma-a r} z, \quad z \rightarrow r-\frac{C}{\sigma-a r} y
$$

system (1) takes the following form

$$
\begin{aligned}
& \dot{x}=-\sigma x+C z+A y z \\
& \dot{y}=R_{a}-y-x z \\
& \dot{z}=-z+x y
\end{aligned}
$$

with

$$
C>0, \quad R_{a}=\frac{r(\sigma-a r)}{C}>0, \quad A=\frac{C^{2} a}{(\sigma-a r)^{2}}>0 .
$$

System (3) was suggested by Glukhovsky and Dolghansky [48], and describes convective fluid motion in an ellipsoidal rotating cavity, which can be interpreted as one of the models of ocean flows (see Appendix A for a description of this problem).

In [99], system (1) was obtained as a linear transformation of the Rabinovich system [146]. It describes interactions between waves in plasma $[140,146]$. Additionally, system (1) describes the following physical processes [99]: a rigid body rotation in a 
resisting medium, the forced motion of a gyrostat, a convective motion in harmonically oscillating horizontal fluid layer, and Kolmogorov flow. Systems (1) and (3) are interesting because of the discovery of chaotic attractors in their phase spaces. Moreover, system (1) was used to describe the specific mechanism of transition to chaos in low-dimensional dynamical systems (gluing bifurcations) [3].

For system (1) with $\sigma= \pm a r,[44]$ contains a detailed analysis of equilibria stability and the asymptotic behavior of trajectories, and a derivation of the parameter values for which the system is integrable. Other researchers have also considered the analytical and numerical analysis of some extensions of system (1) [121,133].

Further, following [48], we consider system (1) with

$$
b=1, \quad a>0, \quad r>0, \quad \sigma>a r .
$$

\subsection{Classical scenario of the transition to chaos}

For the Lorenz system [155], the following classical scenario of transition to chaos is known. Suppose that $\sigma$ and $b$ are fixed (let us consider the classical parameters $\sigma=10, b=8 / 3)$, and that $r$ varies. Then, as $r$ increases, the phase space of the Lorenz system is subject to the following sequence of bifurcations. For $0<r<1$, there is globally asymptotically stable zero equilibrium $S_{0}$. For $r>1$, equilibrium $S_{0}$ is a saddle, and a pair of symmetric equilibria $S_{1,2}$ appears. For $1<r<r_{h} \approx 13.9$, the separatrices $\Gamma_{1,2}$ of equilibria $S_{0}$ are attracted to the equilibria $S_{1,2}$. For $r=r_{h} \approx 13.9$, the separatrices $\Gamma_{1,2}$ form two homoclinic trajectories of equilibria $S_{0}$ (homoclinic butterfly). For $r_{h}<r<r_{c} \approx 24.06$, the separatrices $\Gamma_{1}$ and $\Gamma_{2}$ tend to $S_{2}$ and $S_{1}$, respectively. For $r_{c}<r$, the separatrices $\Gamma_{1,2}$ are attracted to a self-excited attractor (see, e.g., [155,174]). For $r>r_{a}$, the equilibria $S_{1,2}$ become unstable. Finally, $r=28$ corresponds to the classical self-excited Lorenz attractor (see Fig. (1)).

Furthermore, it has been shown that system (1) follows a similar scenario of transition to chaos. However, a substantial distinction of this scenario is the presence of hidden chaotic attractor in the phase space of system (1) for certain parameters values [91].

Let us determine the stationary points of system (1). We can show that for positive parameters, if $r<1$, system (1) has a unique equilibrium $\mathrm{S}_{0}=(0,0,0)$, which is globally asymptotically Lyapunov stable [19]. If $r>1$, then (1) possesses three equilibria: a saddle $S_{0}=(0,0,0)$ and symmetric (with respect to $z=0$ ) equilibria

$$
\mathrm{S}_{1,2}=\left( \pm x_{1}, \pm y_{1}, z_{1}\right),
$$

where

and

$$
x_{1}=\frac{\sigma \sqrt{\xi}}{\sigma+a \xi}, \quad y_{1}=\sqrt{\xi}, \quad z_{1}=\frac{\sigma \xi}{\sigma 1+a \xi}
$$

$$
\xi=\frac{\sigma}{2 a^{2}}\left[a(r-2)-\sigma+\sqrt{(\sigma-a r)^{2}+4 a \sigma}\right] .
$$

The characteristic polynomial of the Jacobian matrix of system (1) at the point $(x, y, z)$ has the form

$$
\chi(x, y, z)=\lambda^{3}+p_{1}(x, y, z) \lambda^{2}+p_{2}(x, y, z) \lambda+p_{3}(x, y, z),
$$

where

$$
\begin{aligned}
& p_{1}(x, y, z)=\sigma+2, \quad p_{2}(x, y, z)=x^{2}+a y^{2}-a z^{2}+(\sigma+a r) z-r \sigma+2 \sigma+1, \\
& p_{3}(x, y, z)=\sigma x^{2}+a y^{2}-a z^{2}-2 a x y z+(\sigma+a r) x y+(\sigma+a r) z-r \sigma+\sigma .
\end{aligned}
$$




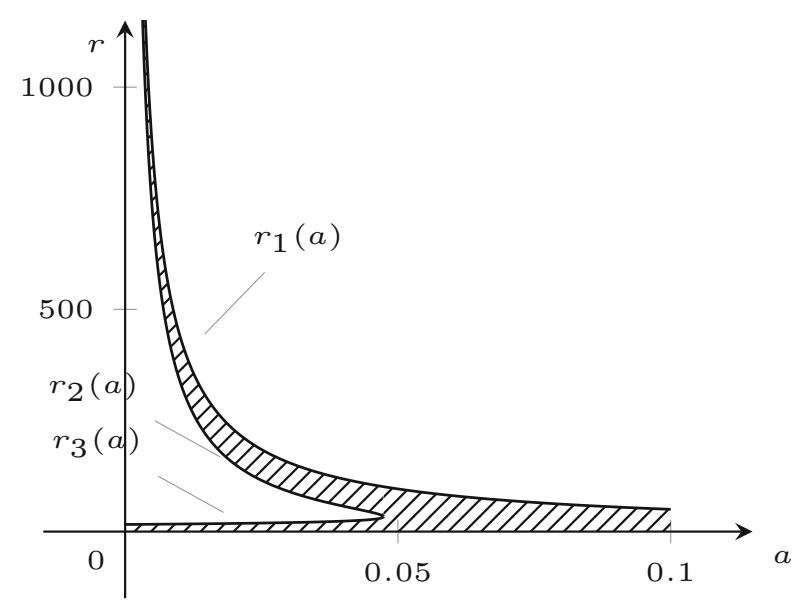

Fig. 4. The stability domain of equilibria $S_{1,2}$ for $\sigma=4$.

Following [48], we let $\sigma=4$ and define the stability domain of equilibria $S_{1,2}$. Using the Routh-Hurwitz criterion we can obtain the following (see Appendix B).

Proposition 1. The equilibria $S_{1,2}$ are stable if

$$
8 a^{2} r^{3}+a(7 a-64) r^{2}+(288 a+128) r+256 a-2048<0 .
$$

The discriminant of the left-hand side of (6) has only one positive real root, $a^{*} \approx$ 0.04735. So the roots of the polynomial in (6) are as follows. For $0<a<a^{*}$, there are three real roots $r_{1}(a)>r_{2}(a)>r_{3}(a)$; for $a=a^{*}$, there are two real roots: $r_{1}(a)$ and $r_{2}(a)=r_{3}(a)$; for $a>a^{*}$, there is one real root $r_{1}(a)$. Thus, for $0<a<a^{*}$, the equilibria $S_{1,2}$ are stable for $r<r_{3}(a)$ and for $r_{2}(a)<r<r_{1}(a)$; and for $a>a^{*}$ the equilibria $S_{1,2}$ are stable for $r<r_{1}(a)$ (see Fig. 4 ).

Consider the problem of the existence of a homoclinic orbit, which is important in bifurcation theory and in scenarios of transition to chaos (see, e.g., [1]). For (1) and (3), we can prove the existence of homoclinic trajectories for the zero saddle equilibrium $S_{0}$ using the fishing principle $[87,96,98,109]$. The fishing principle is based on the construction of a special two-dimensional manifold such that a separatrix of a saddle point intersects or does not intersect the manifold for two different system parameter values. Continuity implies the existence of some intermediate parameter value for which the separatrix touches the manifold. According to the construction, the separatrix must touch a saddle point, so we can numerically localize the birth of a homoclinic orbit. A rigorous description is given in Appendix E.

For $\sigma=4, a=0.0052$, and $r \approx 7.443$ we numerically obtain a homoclinic trajectory (see Fig. 5).

We come now to the study of the limit behaviors of trajectories and attractors. We introduce some rigorous notions of a dynamical system and attractor and discuss the connection with the notions of self-excited and hidden attractors from a computational perspective.

\section{Definitions of attractors}

\subsection{Dynamical systems and ordinary differential equations}

Consider an autonomous system of the differential equations

$$
\dot{\mathrm{x}}=\mathrm{f}(\mathrm{x}), \quad t \in \mathbb{R}, \mathrm{x} \in \mathbb{R}^{n},
$$




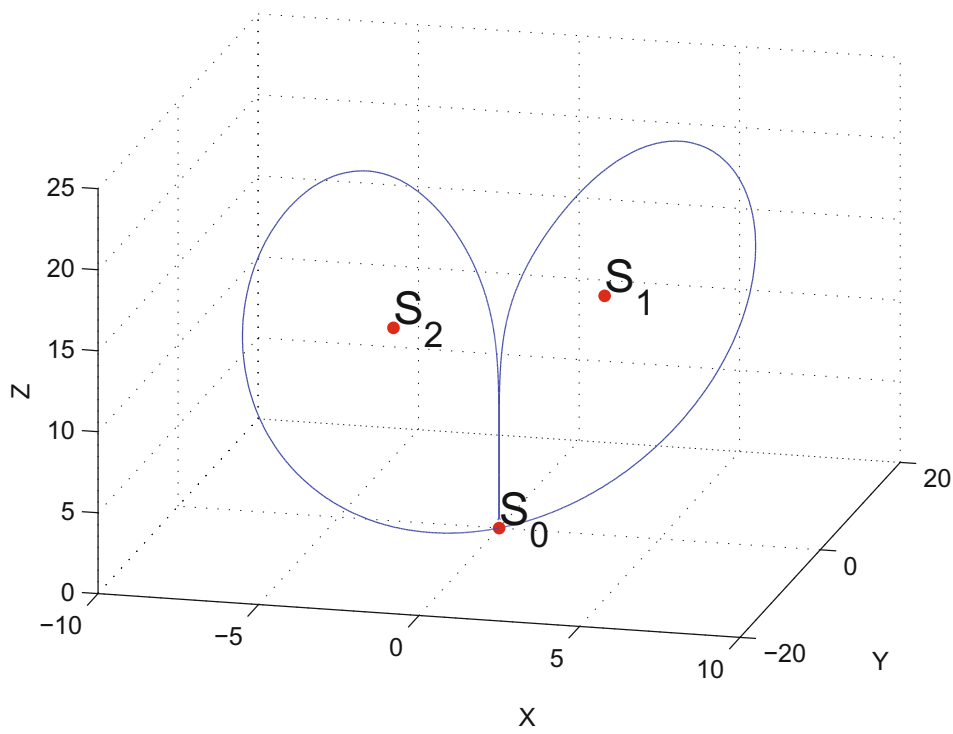

Fig. 5. Separatrices of the saddle $S_{0}=(0,0,0)$ of system (1) for $\sigma=4, a=0.0052 \ldots$, and $r \approx 7.443$.

where $\mathrm{f}: \mathbb{R}^{n} \rightarrow \mathbb{R}^{n}$ is a continuous vector-function that satisfies a local Lipschitz condition in $\mathbb{R}^{n}$. The Picard theorem (see, e.g., $[35,49]$ ) for a local Lipschitz condition on the function $\mathrm{f}$ implies that, for any $\mathrm{x}_{0} \in \mathbb{R}^{n}$, there exists a unique solution $\mathrm{x}\left(t, \mathrm{x}_{0}\right)$ to differential Eq. (7) with the initial data $\mathrm{x}\left(t_{0}, \mathrm{x}_{0}\right)=\mathrm{x}_{0}$, which is given on a certain finite time interval: $t \in I \subset \mathbb{R}$. The theorem regarding the continuous dependence on initial data $[35,49]^{3}$ implies that the solution $\mathrm{x}\left(t, \mathrm{x}_{0}\right)$ continuously depends on $\mathrm{x}_{0}$.

To study the limit behavior of trajectories and compute the limit values, characterizing trajectories, we consider the solutions of (7) for $t \rightarrow+\infty$ or $t \rightarrow \pm \infty$. For arbitrary quadratic systems, the existence of solutions for $t \in\left[t_{0},+\infty\right)$ does not generally imply the existence of solutions for $t \in\left(-\infty, t_{0}\right]$ (see the classical one-dimensional example $\dot{x}=x^{2}$ or multidimensional examples from the work on the completeness of quadratic polynomial systems [47]). It is known that if $\mathrm{f}$ is continuously differentiable ( $\mathrm{f} \in C^{1}$ ), then $\mathrm{f}$ is locally Lipschitz continuous in $\mathbb{R}^{n}$ (see, e.g., [52]). Additionally, if $\mathrm{f}$ is locally Lipschitz continuous, then for any $\mathrm{x}_{0} \in \mathbb{R}^{n}$ the solution $\mathrm{x}\left(\cdot, \mathrm{x}_{0}\right): I \rightarrow \mathbb{R}^{n}$ exists on maximal time interval $I=\left(t_{-}, t_{+}\right) \in \mathbb{R}$, where $-\infty \leq t_{-}<t_{+} \leq+\infty$. If $t_{+}<+\infty$, then $\left\|\mathrm{x}\left(t, \mathrm{x}_{0}\right)\right\| \rightarrow \infty$ for $t \rightarrow t_{+}$, and if $t_{-}>-\infty$, then $\left\|\mathrm{x}\left(t, \mathrm{x}_{0}\right)\right\| \rightarrow \infty$ for $t \rightarrow t_{-}$(see, e.g., [161]). This implies that a solution of (7) is continuous if it remains bounded. For convenience, we introduce a set of time values $\mathbb{T} \in\left\{\mathbb{R}, \mathbb{R}_{+}\right\}$. The existence and uniqueness of solutions of (1) for all $t \in \mathbb{T}$ can be provided, for example, by a global Lipschitz condition.

Another effective method for studying the boundedness of solutions for all $t \in \mathbb{T}$ is to construct a Lyapunov function.

If the existence and uniqueness conditions for all $t \in \mathbb{T}$ are satisfied, then: 1) the solution of (7) satisfies the group property $([35,49])$

$$
\mathrm{x}\left(t+s, \mathrm{x}_{0}\right)=\mathrm{x}\left(t, \mathrm{x}\left(s, \mathrm{x}_{0}\right)\right), \quad \forall t, s \in \mathbb{T},
$$

\footnotetext{
3 Similar theorems on the existence, unicity, and continuous dependence on the initial data for solutions of system with the discontinuous right-hand side are considered in $[64,172]$.
} 
and 2) $\mathrm{x}(\cdot, \cdot): \mathbb{T} \times \mathbb{R}^{n} \rightarrow \mathbb{R}^{n}$ is a continuous mapping according to the theorem of the continuous dependence of the solution on the initial data. Thus, if the solutions of (7) exist and satisfy (8) for all $t \in \mathbb{T}$, the system generates a dynamical system [17] on the phase space $\left(\mathbb{R}^{n},\|\cdot\|\right)$. Here $\|\mathrm{x}\|=\sqrt{x_{1}^{2}+\cdots+x_{n}^{2}}$ is an Euclidean norm of the vector $\mathrm{x}=\left(x_{1}, \ldots, x_{n}\right) \in \mathbb{R}^{n}$, which generates a metric on $\mathbb{R}^{n}$. We abbreviate "dynamical system generated by a differential equation" to "dynamical system". Because the initial time is not important for dynamical systems, without loss of generality we consider

$$
x\left(t, x_{0}\right): x\left(0, x_{0}\right)=x_{0} .
$$

Consider system (1). Its right-hand side is continuously differentiable in $\mathbb{R}^{n}$, which means that it is locally Lipschitz continuous in $\mathbb{R}^{n}$ (but not globally Lipschitz continuous). Analogous with the results for the Lorenz system $[36,126]$, we can prove that the solutions of (1) exist for all $t \in \mathbb{R}$, i.e. system (1) is invertible. For this purpose, we can use the Lyapunov function (Appendix C)

$$
V(x, y, z)=\frac{1}{2}\left(x^{2}+y^{2}+(a+1)\left(z-\frac{\sigma+r}{a+1}\right)^{2}\right) \geq 0 .
$$

Then, system (1) generates a dynamical system and we can study its limit behavior and attractors.

\subsection{Classical definitions of attractors}

The notion of an attractor is connected with investigations of the limit behavior of the trajectories of dynamical systems. We define attractors as follows $[9,19,32,34,82$, $83,95,160]$.

Definition 2. A set $K$ is said to be positively invariant for a dynamical system if

$$
\mathrm{x}(t, K) \subset K, \forall t \geq 0,
$$

and to be invariant if

$$
\mathrm{x}(t, K)=K, \forall t \geq 0,
$$

where $\mathrm{x}(t, K)=\left\{\mathrm{x}\left(t, \mathrm{x}_{0}\right) \mid \mathrm{x}_{0} \in K, t \geq 0\right\}$.

Property 1. Invariant set $K$ is said to be locally attractive for a dynamical system if, for a certain $\varepsilon$-neighborhood $K(\varepsilon)$ of set $K$,

$$
\lim _{t \rightarrow+\infty} \rho\left(K, \mathrm{x}\left(t, \mathrm{x}_{0}\right)\right)=0, \quad \forall \mathrm{x}_{0} \in K(\varepsilon) .
$$

Here $\rho(K, \mathrm{x})$ is a distance from the point $\mathrm{x}$ to the set $K$, defined as

$$
\rho(K, \mathrm{x})=\inf _{\mathrm{z} \in K}\|\mathrm{z}-\mathrm{x}\|
$$

and $K(\varepsilon)$ is a set of points $\mathrm{x}$ for which $\rho(K, \mathrm{x})<\varepsilon$.

Property 2. Invariant set $K$ is said to be globally attractive for dynamical system if

$$
\lim _{t \rightarrow+\infty} \rho\left(K, \mathrm{x}\left(t, \mathrm{x}_{0}\right)\right)=0, \quad \forall \mathrm{x}_{0} \in \mathbb{R}^{n} .
$$


Property 3. Invariant set $K$ is said to be uniformly locally attractive for a dynamical system if for a certain $\varepsilon$-neighborhood $K(\varepsilon)$, any number $\delta>0$, and any bounded set $B$, there exists a number $t(\delta, B)>0$ such that

$$
\mathrm{x}(t, B \cap K(\varepsilon)) \subset K(\delta), \quad \forall t \geq t(\delta, B) .
$$

Here

$$
\mathrm{x}(t, B \cap K(\varepsilon))=\left\{\mathrm{x}\left(t, \mathrm{x}_{0}\right) \mid \mathrm{x}_{0} \in B \cap K(\varepsilon)\right\} .
$$

Property 4. Invariant set $K$ is said to be uniformly globally attractive for a dynamical system if, for any number $\delta>0$ and any bounded set $B \subset \mathbb{R}^{n}$, there exists a number $t(\delta, B)>0$ such that

$$
\mathrm{x}(t, B) \subset K(\delta), \quad \forall t \geq t(\delta, B) .
$$

Definition 3. For a dynamical system, a bounded closed invariant set $K$ is:

(1) an attractor if it is a locally attractive set (i.e., it satisfies Property 1);

(2) a global attractor if it is a globally attractive set (i.e., it satisfies Property 2);

(3) a B-attractor if it is a uniformly locally attractive set (i.e., it satisfies Property 3); or

(4) a global B-attractor if it is a uniformly globally attractive set (i.e., it satisfies Property 4).

Remark 1. In the definition of an attractor we assume closeness for the sake of uniqueness. This is because the closure of a locally attractive invariant set $K$ is also a locally attractive invariant set (for example, consider an attractor with excluded one of the embedded unstable periodic orbits). The closeness property is sometimes omitted from the attractor definition (see, e.g., [8]). Additionally, the boundedness property is sometimes omitted (see, e.g., [29]). For example, a global attractor in a system describing a pendulum motion is not bounded in the phase space $\mathbb{R}^{2}$ (but it is bounded in the cylindrical phase space). Unbounded attractors are considered for nonautonomous systems in the extended phase space. Note that if a dynamical system is defined for $t \in \mathbb{R}$, then a locally attractive invariant set only contains the whole trajectories, i.e. if $x_{0} \in K$, then $x\left(t, x_{0}\right) \in K$ for $\forall t \in \mathbb{R}$ (see [32]).

Remark 2. The definition considered here implies that a global B-attractor is also a global attractor. Consequently, it is rational to introduce the notion of a minimal global attractor (or minimal attractor) [32,34]. This is the smallest bounded closed invariant set that possesses Property 2 (or Property 1). Further, the attractors (global attractors) will be interpreted as minimal attractors (minimal global attractors).

Definition 4. For an attractor $K$, the basin of attraction is a set $B(K) \subset \mathbb{R}^{n}$ such that

$$
\lim _{t \rightarrow+\infty} \rho\left(K, \mathrm{x}\left(t, \mathrm{x}_{0}\right)\right)=0, \quad \forall \mathrm{x}_{0} \in B(K)
$$

Remark 3. From a computational perspective, it is not feasible to numerically check Property 1 for all initial states of the phase space of a dynamical system. A natural generalization of the notion of an attractor is consideration of the weaker attraction requirements: almost everywhere or on a set of the positive Lebesgue measure (see, e.g., [128]). See also trajectory attractors [30,33,151]. To distinguish an artificial computer generated chaos from a real behavior of the system one can consider the shadowing property of the system (see, e.g., the survey in [141]).

We can typically see an attractor (or global attractor) in numerical experiments. The notion of a B-attractor is mostly used in the theory of dimensions, where we consider invariant sets covered by balls. The uniform attraction requirement in Property 3 


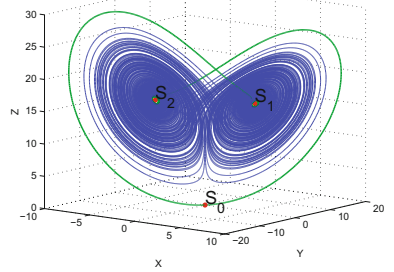

(a) Two trajectories with symmetric initial data near $S_{0}$

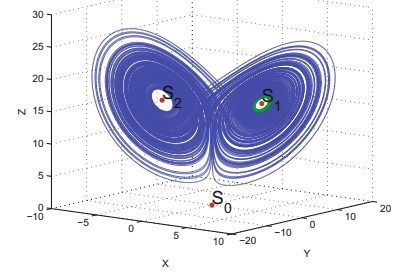

(b) A trajectory with initial data near $S_{1}$

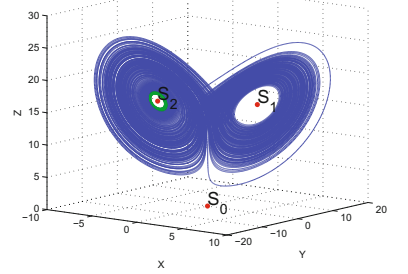

(c) A trajectory with initial data near $S_{2}$

Fig. 6. Self-excited attractor of system (1) for $r=17, \sigma=4$, and $a=0.0052$, computed from different initial points.

implies that a global B-attractor involves a set of stationary points $(\mathcal{S})$ and the corresponding unstable manifolds $W^{u}(\mathcal{S})=\left\{\mathrm{x}_{0} \in \mathbb{R}^{n} \mid \lim _{t \rightarrow-\infty} \rho\left(\mathcal{S}, \mathrm{x}\left(t, \mathrm{x}_{0}\right)\right)=0\right\}$ (see, e.g., $[32,34])$. The same is true for B-attractor if the considered neighborhood $K(\varepsilon)$ in Property 3 contains some of the stationary points from $\mathcal{S}$. From a computational perspective, numerically checking Property 3 is also difficult. Therefore if the basin of attraction involves unstable manifolds of equilibria, then computing the minimal attractor and the unstable manifolds that are attracted to it may be regarded as an approximation of B-attractor. For example, consider the visualization of the classical Lorenz attractor from the neighborhood of the zero saddle equilibria. Note that a minimal global attractor involves the set $\mathcal{S}$ and its basin of attraction involves the set $W^{u}(\mathcal{S})$. Various analytical-numerical methods for computing attractors and their basins of attraction can be found in, for example, [7,39,46,132,164,177].

\section{Self-excited attractor localization}

In [48] system (3) with $\sigma=4$ was studied. Consider the following parameters for system (1)

$$
\sigma=4, \quad a=0.0052
$$

According to Proposition 1 , if $r_{1} \approx 16.4961242 \ldots<r<r_{2} \approx 690.6735024$, the equilibria $S_{1,2}$ of system (1) become (unstable) saddle-focuses. For example, if $r=17$, the eigenvalues of the equilibria of system (1) are the following

$$
\begin{array}{ccc}
S_{0}: & 5.8815, \quad-1, & -10.8815 \\
S_{1,2}: & 0.0084 \pm 4.5643 \mathrm{i}, & -6.0168
\end{array}
$$

and there is a self-excited chaotic attractor in the phase space of system (1). We can easily visualize this attractor (Fig. 6) using the standard computational procedure with initial data in the vicinity of one of the equilibria $S_{0,1,2}$ on the corresponding unstable manifolds. To improve the approximation of the attractor one can consider its neighborhood and compute trajectories from a grid of points in this neighborhood.

\section{Hidden attractor localization}

We need a special numerical method to localize the hidden attractor of system (1), because the basin of attraction does not intersect the small neighborhoods of the 


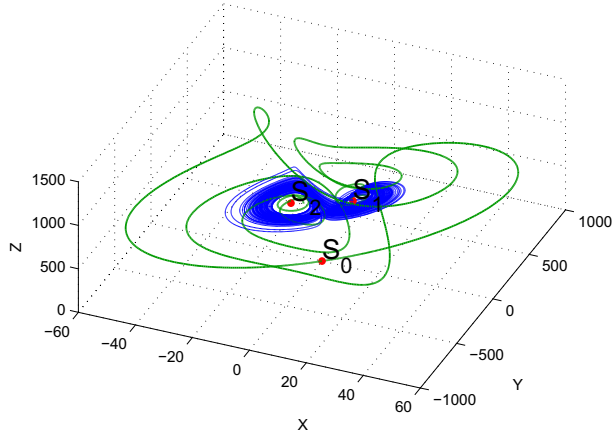

(a) $P_{0}: r=687.5$. Attractor (blue), B-attractor (green, blue and red)

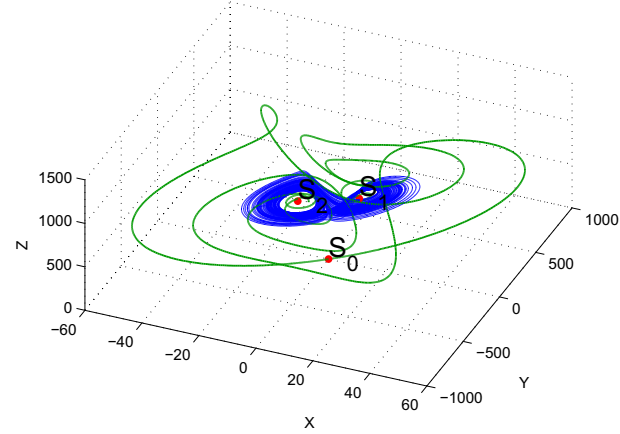

(b) $r=690$. Attractor (blue), B-attractor (green. blue and red)

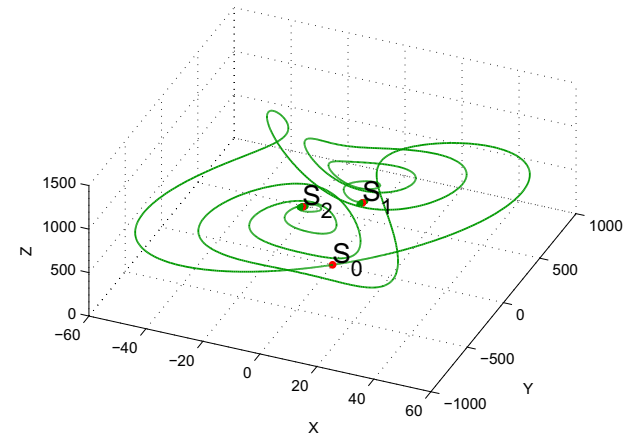

(c) $P_{1}: \quad r=700$. B-attractor (green and red)

Fig. 7. B-attractor of system (1) for fixed $\sigma=4, a=0.0052$, and various $r$.

unstable manifolds of the equilibria. One effective method for the numerical localization of hidden attractors is based on a homotopy and numerical continuation. We construct a sequence of similar systems such that the initial data for numerically computing the oscillating solution (starting oscillation) can be obtained analytically for the first (starting) system. For example, it is often possible to consider a starting system with a self-excited starting oscillation. Then we numerically track the transformation of the starting oscillation while passing between systems.

In a scenario of transition to chaos in dynamical system there is typically a parameter $\lambda \in\left[a_{1}, a_{2}\right]$, the variation of which gives the scenario. We can also artificially introduce the parameter $\lambda$, let it vary in the interval $\left[a_{1}, a_{2}\right]$ (where $\lambda=a_{2}$ corresponds to the initial system), and choose a parameter $a_{1}$ such that we can analytically or computationally find a certain nontrivial attractor when $\lambda=a_{1}$ (often this attractor has a simple form, e.g., periodic). That is, instead of analyzing the scenario of a transition into chaos, we can synthesize it. Further, we consider the sequence $\lambda_{j}, \lambda_{1}=a_{1}, \lambda_{m}=a_{2}, \lambda_{j} \in\left[a_{1}, a_{2}\right]$ such that the distance between $\lambda_{j}$ and $\lambda_{j+1}$ is sufficiently small. Then we numerically investigate changes to the shape of the attractor obtained for $\lambda_{1}=a_{1}$. If the change in $\lambda$ (from $\lambda_{j}$ to $\lambda_{j+1}$ ) does not cause a loss of the stability bifurcation of the considered attractor, then the attractor for $\lambda_{m}=a_{2}$ (at the end of procedure) is localized.

Let us construct a line segment on the plane $(a, r)$ that intersects a boundary of the stability domain of the equilibria $S_{1,2}$ (see Fig. 8). We choose the point $P_{1}(r=700, a=0.0052)$ as the end point of the line segment. The eigenvalues for 


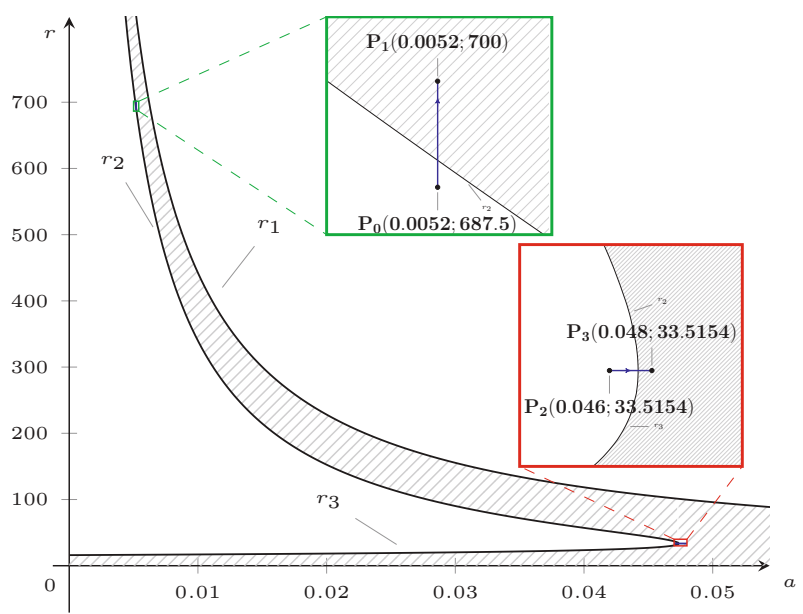

Fig. 8. Paths $\left[P_{0}, P_{1}\right]$ and $\left[P_{2}, P_{3}\right]$ in the plane of parameters $\{a, r\}$ used in the continuation procedure.

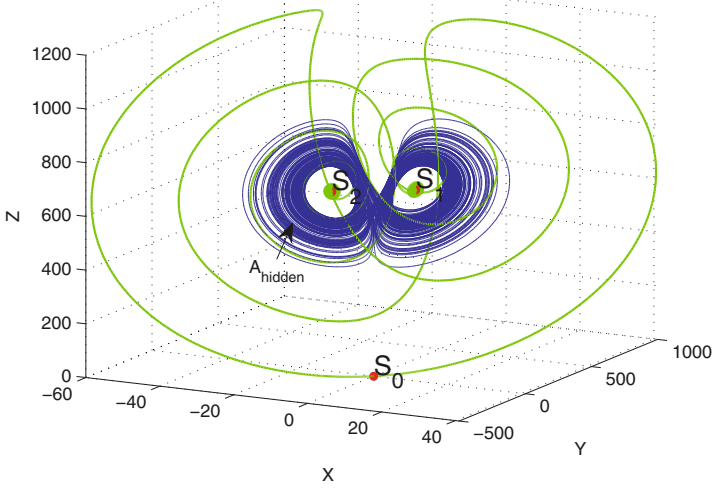

Fig. 9. Hidden attractor (blue) coexist with B-attractor (green outgoing separatrix of the saddle $S_{0}$ attracted to the red equilibria $\left.S_{1,2}\right)$.

the equilibria of system (1) that correspond to the parameters $P_{1}$ are the following:

$$
\begin{array}{ccc}
S_{0}: & 50.4741, \quad-1, \quad-55.4741, \\
S_{1,2}: & -0.1087 \pm 10.4543 \mathrm{i}, \quad-5.7826 .
\end{array}
$$

This means that the equilibria $S_{1,2}$ become stable focus-nodes. Now we choose the point $P_{0}(r=687.5, a=0.0052)$ as the initial point of the line segment. This point corresponds to the parameters for which system (1) has a self-excited attractor, which can be computed using the standard computational procedure. Then we choose a sufficiently small partition step for the line segment and compute a chaotic attractor in the phase space of system (1) at each iteration of the procedure. The last computed point at each step is used as the initial point for the computation at the next step (the computation time must be sufficiently large).

In our experiment the length of the path was 2.5 and there were 6 iterations. Here for the selected path and partition, we can visualize a hidden attractor of system (1) (see Fig. 9). The results of continuation procedure are given in [91]. 
Note that the choice of path and its partitions in the continuation procedure is not trivial. For example, a similar procedure does not lead to a hidden attractor for the following path on the plane $(a, r)$. Consider $r=33.51541181, a=0.04735056 \ldots$ (the rightmost point on the stability domain), and take a starting point $P_{2}: r=$ 33.51541181, $a=0.046$ near it (Fig. 8). If we use the partition step 0.001 , then there are no hidden attractors after crossing the boundary of the stability domain. For example, if the end point is $P_{3}: r=33.51541181, a=0.048$, there is no chaotic attractor but only trivial attractors (the equilibria $S_{1,2}$ ).

\section{Analytical localization of global attractor via Lyapunov functions}

In the previous sections, we considered the numerical localization of various selfexcited and hidden attractors of system (1). It is natural to question if these attractors (or the union of attractors) are global (in the sense of Definition 3) or if other coexisting attractors can be found.

The dissipativity property is important when proving the existence of a bounded global attractor for a dynamical system and gives an analytical localization of the global attractor in the phase space. The dissipativity of a system, on one hand, proves that there are no trajectories that tend to infinity as $t \rightarrow+\infty$ in the phase space and, on the other hand, can be used one to determine the boundaries of the domain that all trajectories enter within a finite time.

Definition 5. A set $B_{0} \subset \mathbb{R}^{n}$ is said to be absorbing for dynamical system (7) if for any $\mathrm{x}_{0} \in \mathbb{R}^{n}$ there exists $T=T\left(\mathrm{x}_{0}\right)$ such that $\mathrm{x}\left(t, \mathrm{x}_{0}\right) \in B_{0}$ for any $t \geq T$.

Note that the trajectory $\mathrm{x}\left(t, \mathrm{x}_{0}\right)$ with $\mathrm{x}_{0} \in B_{0}$ may leave $B_{0}$ for only a finite time before it returns and stays inside for $t \geq T$.

Remark 4. In [116] the ball $B_{R}=\left\{\mathrm{x} \in \mathbb{R}^{n}:|\mathrm{x}|<R\right\}$ was regarded as an absorbing set. In this case, if there exists $R>0$ such that

$$
\limsup _{t \rightarrow \infty}\left|\mathrm{x}\left(t, \mathrm{x}_{0}\right)\right|<R, \quad \text { for any } \mathrm{x}_{0} \in \mathbb{R}^{n}
$$

then it is said that a dynamical system is dissipative in the sense of Levinson . $R$ is called a radius of dissipativity ${ }^{4}$.

Definition 6 ([32,34]). Dynamical system (7) is called (pointwise) dissipative ${ }^{5}$ if it possesses a bounded absorbing set.

Theorem 1 ([32,34]). If dynamical system (7) is dissipative, then it possesses a global B-attractor.

We can effectively prove dissipativity by constructing the Lyapunov function $[92,173]$. Consider a sufficient condition of dissipativity for system (7).

\footnotetext{
${ }^{4}$ Because any greater radius also satisfies the definition, the minimal $R$ is of interest for the problems of attractor localization and definition of ultimate bound.

5 Together with the notions of an absorbing set and dissipative system, $[19,83]$ also considered the definitions of a $B$-absorbing set and a $B$-dissipative system (uniform convergence of trajectories to the corresponding B-absorbing set). It is known [19] that if a dynamical system given on $\left(\mathbb{R}^{n},\|\cdot\|\right)$ is dissipative, then it is also B-dissipative.
} 


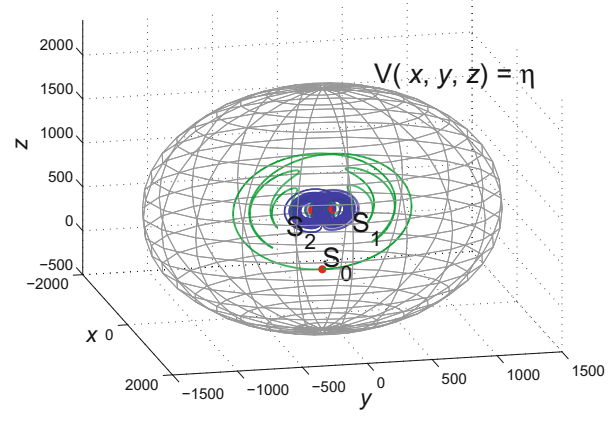

(a) Absorbing set (grey), self-excited attractor (blue), and global B-attractor (blue, green and red) for system (1) with the parameters $r=687.5, \sigma=4$, and $a=$ 0.0052. $\eta \approx 238541.3$.

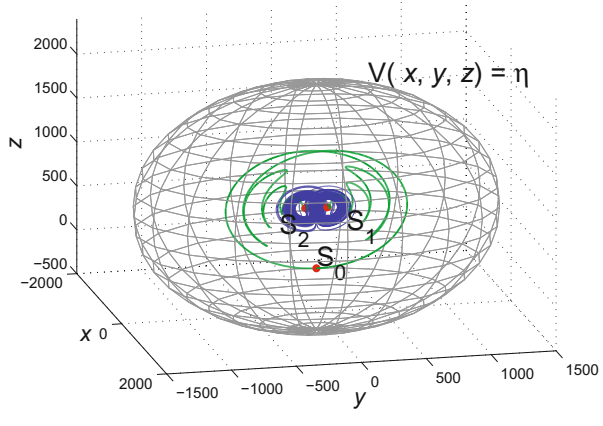

(b) Absorbing set (grey), hidden attractor (blue), and global B-attractor (blue, green and red) for system (1) with the parameters $r=700, \sigma=4$, and $a=0.0052$. $\eta \approx 247230.5$.

Fig. 10. Absorbing sets for system (1).

Theorem $2([\mathbf{1 4 9}, \mathbf{1 7 1}])$. Suppose that there exists continuously differentiable function $V(\mathrm{x}): \mathbb{R}^{n} \rightarrow \mathbb{R}$, possessing the following properties.

(1) $\lim _{|\mathrm{x}| \rightarrow \infty} V(\mathrm{x})=+\infty$, and

(2) there exist numbers $R$ and $\varkappa$ such that for any solution $\mathrm{x}\left(t, \mathrm{x}_{0}\right)$ of system (7), the condition $\left|\mathrm{x}\left(t, \mathrm{x}_{0}\right)\right|>R$ implies that $\dot{V}\left(\mathrm{x}\left(t, \mathrm{x}_{0}\right)\right) \leq-\varkappa$.

\section{Then}

(a) any solution $\mathrm{x}\left(t, \mathrm{x}_{0}\right)$ to ( 7$)$ exists at least on $[0,+\infty)$, so system (7) generates a dynamical system for any $t \geq 0$ and $\mathrm{x}_{0} \in \mathbb{R}^{n}$; and

(b) if $\eta>0$ is such that $B_{0}=\left\{\mathrm{x} \in \mathbb{R}^{n} \mid V(\mathrm{x}) \leq \eta\right\} \supset\left\{\mathrm{x} \in \mathbb{R}^{n} \mid\|\mathrm{x}\|<R\right\}$, then $B_{0}$ is a compact absorbing set of dynamical system (7).

More general theorems, connected with the application of the Lyapunov functions to the proof of dissipativity for dynamical systems can be found in $[101,147]$.

It is known that the Lorenz system is dissipative (it is sufficient to choose the Lyapunov function $\left.V(x, y, z)=\frac{1}{2}\left(x^{2}+y^{2}+(z-r-\sigma)^{2}\right)\right)$. However, for example, one of the Rossler systems is not dissipative in the sense of Levinson [115] because the outgoing separatrix is unbounded. In the general case, there is an art in the construction of Lyapunov functions which prove dissipativity.

Lemma 1. Dynamical system (1) is dissipative.

The proof is based on Lyapunov function $V$ from (9) (see Appendix C). If $R, \eta$ are chosen as in the proof of Theorem 1, Appendix C, then dynamical system (1) has a compact absorbing set

$$
B_{0}=\left\{(x, y, z): V(x, y, z)=\frac{1}{2}\left(x^{2}+y^{2}+(a+1)\left(z-\frac{\sigma+r}{a+1}\right)^{2}\right) \leq \eta\right\} .
$$

For example, for $\sigma>1, r>1, a<1$ we can choose $R=\frac{\sigma+r}{a+1}$ and $\eta=2(a+$ 1) $R^{2}$ (see Fig. 10). Note that for system (1) the ellipsoidal absorbing set $B_{0}$ can be improved using special additional transformations and Yudovich's theorem (see, e.g., [14]), similarly to [93] for the Lorenz system. 
There is also a cylindrical positively invariant set for system (1) [87],

$$
C=\left\{|x| \leq r+\frac{2 a}{\sigma} r^{2}, \quad y^{2}+(z-r)^{2} \leq r^{2}\right\}
$$

because

$$
\left(y^{2}+(z-r)^{2}\right)^{\bullet} \leq-\left(y^{2}+(z-r)^{2}\right)+r^{2}<0 \quad \forall x,|y|>r \text { or }|z|>2 r
$$

and

$$
|x|^{\bullet} \leq-\sigma|x|+\sigma|y|+a|y||z|<0 \quad|y| \leq r,|z| \leq 2 r,|x|>r+2 a r^{2} / \sigma .
$$

Thus, as for the Lorenz system [100], we obtain three different estimates of the attractor: the ball $B_{R}$, ellipse $B_{0}$, and cylinder $C$.

\section{Upper estimate of the Lyapunov dimension of attractor}

\subsection{Lyapunov exponents and Lyapunov dimension}

Suppose that the right-hand side of system (7) is sufficiently smooth, and consider a linearized system along a solution $\mathrm{x}\left(t, \mathrm{x}_{0}\right)$. We have

$$
\dot{\mathrm{u}}=J\left(\mathrm{x}\left(t, \mathrm{x}_{0}\right)\right) \mathrm{u}, \quad \mathrm{u} \in \mathbb{R}^{n}, t \in \mathbb{R}_{+},
$$

where

$$
J\left(\mathrm{x}\left(t, \mathrm{x}_{0}\right)\right)=\left[\left.\frac{\partial f_{i}(\mathrm{x})}{\partial \mathrm{x}_{j}}\right|_{\mathrm{x}=\mathrm{x}\left(t, \mathrm{x}_{0}\right)}\right]
$$

is the $(n \times n)$ Jacobian matrix evaluated along the trajectory $\mathrm{x}\left(t, \mathrm{x}_{0}\right)$ of system $(7)$. A fundamental matrix $X\left(t, \mathrm{x}_{0}\right)$ of linearized system (11) is defined by the variational equation

$$
\dot{X}\left(t, \mathrm{x}_{0}\right)=J\left(\mathrm{x}\left(t, \mathrm{x}_{0}\right)\right) X\left(t, \mathrm{x}_{0}\right) .
$$

We typically set $X\left(0, \mathrm{x}_{0}\right)=I_{n}$, where $I_{n}$ is the identity matrix. Then $\mathrm{u}\left(t, \mathrm{u}_{0}\right)=$ $X\left(t, \mathrm{x}_{0}\right) \mathrm{u}_{0}$. In the general case, $\mathrm{u}\left(t, \mathrm{u}_{0}\right)=X\left(t, \mathrm{x}_{0}\right) X^{-1}\left(0, \mathrm{x}_{0}\right) \mathrm{u}_{0}$. Note that if a solution of nonlinear system (7) is known, then we have

$$
X\left(t, \mathrm{x}_{0}\right)=\frac{\partial \mathrm{x}\left(t, \mathrm{x}_{0}\right)}{\partial \mathrm{x}_{0}}
$$

Two well-known definitions of Lyapunov exponents are the upper bounds of the exponential growth rate of the norms of linearized system solutions (LCEs) [124] and the upper bounds of the exponential growth rate of the singular values of fundamental matrix of linearized system (LEs) [131].

Let $\sigma_{1}\left(X\left(t, \mathrm{x}_{0}\right)\right) \geq \cdots \geq \sigma_{n}\left(X\left(t, \mathrm{x}_{0}\right)\right)>0$ denote the singular values of a fundamental matrix $X\left(t, \mathrm{x}_{0}\right)$ (the square roots of the eigenvalues of the matrix $X\left(t, \mathrm{x}_{0}\right)^{*} X\left(t, \mathrm{x}_{0}\right)$ are reordered for each $\left.t\right)$.

Definition 7. The Lyapunov exponents (LEs) at the point $\mathrm{x}_{0}$ are the numbers (or the symbols $\pm \infty$ ) defined by

$$
\mathrm{LE}_{i}\left(\mathrm{x}_{0}\right)=\limsup _{t \rightarrow \infty} \frac{1}{t} \ln \sigma_{i}\left(X\left(t, \mathrm{x}_{0}\right)\right)
$$


LEs are commonly used ${ }^{6}$ in the theories of dynamical systems and attractor dimensions $[11,19,43,55,85,160]$.

Remark 5. The LEs are independent of the choice of fundamental matrix at the point $\mathrm{x}_{0}$ [74] unlike the Lyapunov characteristic exponents (LCEs, see [124]). To determine all possible values of LCEs, we must consider a normal fundamental matrix.

We now define a Lyapunov dimension [62]

Definition 8. A local Lyapunov dimension of a point $\mathrm{x}_{0}$ in the phase space of a dynamical system is as follows: $\operatorname{dim}_{L} \mathrm{x}_{0}=0$ if $\mathrm{LE}_{1}\left(x_{0}\right) \leq 0$ and $\operatorname{dim}_{L} \mathrm{x}_{0}=n$ if $\sum_{i=1}^{n} \operatorname{LE}_{i}^{o}\left(x_{0}\right) \geq 0$, otherwise

$$
\operatorname{dim}_{L} \mathrm{x}_{0}=j\left(\mathrm{x}_{0}\right)+\frac{\mathrm{LE}_{1}\left(\mathrm{x}_{0}\right)+\ldots+\mathrm{LE}_{j}\left(\mathrm{x}_{0}\right)}{\left|\mathrm{LE}_{j+1}\left(\mathrm{x}_{0}\right)\right|}
$$

where $\mathrm{LE}_{1}\left(\mathrm{x}_{0}\right) \geq \ldots \geq \mathrm{LE}_{n}\left(\mathrm{x}_{0}\right)$ are ordered LEs and $j\left(\mathrm{x}_{0}\right) \in[1, n]$ is the smallest natural number $m$ such that

$$
\mathrm{LE}_{1}\left(\mathrm{x}_{0}\right)+\ldots+\mathrm{LE}_{m}\left(\mathrm{x}_{0}\right)>0, \quad \mathrm{LE}_{m+1}\left(\mathrm{x}_{0}\right)<0, \quad \frac{\mathrm{LE}_{1}\left(\mathrm{x}_{0}\right)+\ldots+\mathrm{LE}_{m}\left(\mathrm{x}_{0}\right)}{\left|\mathrm{LE}_{m+1}\left(\mathrm{x}_{0}\right)\right|}<1 .
$$

The Lyapunov dimension of invariant set $K$ of a dynamical system is defined as

$$
\operatorname{dim}_{L} K=\sup _{x_{0} \in K} \operatorname{dim}_{L} x_{0}
$$

Note that, from an applications perspective, an important property of the Lyapunov dimension is the chain of inequalities $[19,55,56]$

$$
\operatorname{dim}_{T} K \leqslant \operatorname{dim}_{H} K \leqslant \operatorname{dim}_{F} K \leqslant \operatorname{dim}_{L} K
$$

Here $\operatorname{dim}_{T} K, \operatorname{dim}_{H} K$, and $\operatorname{dim}_{F} K$ are the topological, Hausdorff, and fractal dimensions of $K$, respectively.

Along with commonly used numerical methods for estimating and computing the Lyapunov dimension, there is an analytical approach that was proposed by Leonov $[19,94,95,97,99,109]$. It is based on the direct Lyapunov method and uses Lyapunovlike functions.

\footnotetext{
6 The LCEs [124] and LEs [131] are "often" equal, e.g., for a "typical" system that satisfies the conditions of Oseledec theorem [131]. However, there are no effective methods for checking Oseledec conditions for a given system: "Oseledec proof is important mathematics, but the method is not helpful in elucidating dynamics" [37, p.118]). For a particular system, LCEs and LEs may be different. For example, for the fundamental matrix $X(t)=\left(\begin{array}{c}1 g(t)-g^{-1}(t) \\ 0 \\ 1\end{array}\right)$ we have the following ordered values: $\mathrm{LCE}_{1}=\max \left(\limsup _{t \rightarrow+\infty} \mathcal{X}[g(t)], \limsup _{t \rightarrow+\infty} \mathcal{X}\left[g^{-1}(t)\right]\right), \mathrm{LCE}_{2}=0 ; \mathrm{LE}_{1,2}=$ $\max , \min \left(\limsup _{t \rightarrow+\infty} \mathcal{X}[g(t)], \limsup _{t \rightarrow+\infty} \mathcal{X}\left[g^{-1}(t)\right]\right)$, where $\mathcal{X}(\cdot)=\frac{1}{t} \log |\cdot|$. Thus, in general, the Kaplan-Yorke (Lyapunov) dimensions based on LEs and LCEs may be different. Note also that positive largest LCE or LE, computed via the linearization of the system along a trajectory, do not necessary imply instability or chaos, because for non-regular linearization there are well-known Perron effects of Lyapunov exponent sign reversal [77,78, 102]. Therefore for the computation of the Lyapunov dimension of an attractor one has to consider a grid of points on the attractor and corresponding local Lyapunov dimensions [81]. More detailed discussion and examples can be found in [74,102].
} 
LEs and the Lyapunov dimension are invariant under linear changes of variables (see, e.g., [74]). Therefore we can apply the linear variable change y $=S \mathrm{x}$ with a nonsingular $n \times n$-matrix $S$. Then system (7) is transformed into

$$
\dot{\mathrm{y}}=S \dot{\mathrm{x}}=S \mathrm{f}\left(S^{-1} \mathrm{y}\right)=\tilde{\mathrm{f}}(\mathrm{y}) .
$$

Consider the linearization along corresponding solution $\mathrm{y}\left(t, \mathrm{y}_{0}\right)=S \mathrm{x}\left(t, S^{-1} \mathrm{x}_{0}\right)$, that is,

$$
\dot{\mathrm{v}}=\tilde{J}\left(\mathrm{y}\left(t, \mathrm{y}_{0}\right)\right) \mathrm{v}, \quad \mathrm{v} \in \mathbb{R}^{n} .
$$

Here the Jacobian matrix is as follows

$$
\tilde{J}\left(\mathrm{y}\left(t, \mathrm{y}_{0}\right)\right)=S J\left(\mathrm{x}\left(t, \mathrm{x}_{0}\right)\right) S^{-1}
$$

and the corresponding fundamental matrix satisfies $Y\left(t, \mathrm{y}_{0}\right)=S X\left(t, \mathrm{x}_{0}\right)$.

For simplicity, let $J(\mathrm{x})=J\left(\mathrm{x}\left(t, \mathrm{x}_{0}\right)\right)$. Suppose that $\lambda_{1}(\mathrm{x}, S) \geqslant \cdots \geqslant \lambda_{n}(\mathrm{x}, S)$ are eigenvalues of the symmetrized Jacobian matrix (18)

$$
\frac{1}{2}\left(S J(\mathrm{x}) S^{-1}+\left(S J(\mathrm{x}) S^{-1}\right)^{*}\right) .
$$

Theorem 3 ([86,97]). Given an integer $j \in[1, n]$ and $s \in[0,1]$, suppose that there are a continuously differentiable scalar function $\vartheta: \mathbb{R}^{n} \rightarrow \mathbb{R}$ and a nonsingular matrix $S$ such that

$$
\lambda_{1}(\mathrm{x}, S)+\cdots+\lambda_{j}(\mathrm{x}, S)+s \lambda_{j+1}(\mathrm{x}, S)+\dot{\vartheta}(\mathrm{x})<0, \forall \mathrm{x} \in K .
$$

Then $\operatorname{dim}_{L} K \leqslant j+s$.

Here $\dot{\vartheta}$ is the derivative of $\vartheta$ with respect to the vector field $\mathrm{f}$ :

$$
\dot{\vartheta}(\mathrm{x})=(\operatorname{grad}(\vartheta))^{*} \mathrm{f}(\mathrm{x}) \text {. }
$$

The introduction of the matrix $S$ can be regarded as a change of the space metric.

Theorem $4([\mathbf{1 9}, \mathbf{9 4}, \mathbf{9 7}, \mathbf{9 9}])$. Assume that there are a continuously differentiable scalar function $\vartheta$ and a nonsingular matrix $S$ such that

$$
\lambda_{1}(\mathrm{x}, S)+\lambda_{2}(\mathrm{x}, S)+\dot{\vartheta}(\mathrm{x})<0, \forall \mathrm{x} \in \mathbb{R}^{n} .
$$

Then any solution of system (7) bounded on $[0,+\infty)$ tends to an equilibrium as $t \rightarrow+\infty$.

Thus, if (21) holds, then the global attractor of system (7) coincides with its stationary set.

Theorems 3 and 4 give the following results for system (1).

Theorem 5. Suppose that $\sigma>1$.

If

$$
\left(r+\frac{\sigma}{a}\right)^{2}<\frac{2(\sigma+1)}{a},
$$

then any solution of system (1) bounded on $[0,+\infty)$ tends to an equilibrium as $t \rightarrow+\infty$.

If

$$
\left(r+\frac{\sigma}{a}\right)^{2}>\frac{2(\sigma+1)}{a}
$$

then

$$
\operatorname{dim}_{L} K \leqslant 3-\frac{2(\sigma+2)}{\sigma+1+\sqrt{(\sigma-1)^{2}+a\left(\frac{\sigma}{a}+r\right)^{2}}} .
$$


Proof. We use the matrix

$$
S=\left(\begin{array}{rrr}
-a^{-\frac{1}{2}} & 0 & 0 \\
0 & 1 & 0 \\
0 & 0 & 1
\end{array}\right)
$$

Then the eigenvalues of the corresponding matrix (19) are the following

$$
\begin{aligned}
\lambda_{2} & =-1 \\
\lambda_{1,3} & =-\frac{\sigma+1}{2} \pm \frac{1}{2}\left[(\sigma-1)^{2}+a\left(2 z-\frac{\sigma+a r}{a}\right)^{2}\right]^{\frac{1}{2}} .
\end{aligned}
$$

To check property (20) of Theorem 3 and property (21) of Theorem 4, we can consider the Lyapunov-like function

$$
\vartheta(x, y, z)=\frac{2(1-s) V(x, y, z)}{\left[(\sigma-1)^{2}+a\left(\frac{\sigma}{a}+r\right)^{2}\right]^{\frac{1}{2}}},
$$

where

$$
V(x, y, z)=\frac{\gamma}{\sigma} x^{2}+\gamma y^{2}+\gamma\left(1+\frac{a}{\sigma}\right) z^{2}-2 \gamma(r-1) z, \quad \gamma=\frac{\sigma+a r}{2(r-1)} .
$$

Finally, for system (1) with given $S$ and $\vartheta$, if condition (23) is satisfied and

$$
s>\frac{-(\sigma+3)+\sqrt{(\sigma-1)^{2}+a\left(\frac{\sigma}{a}+r\right)^{2}}}{\sigma+1+\sqrt{(\sigma-1)^{2}+a\left(\frac{\sigma}{a}+r\right)^{2}}},
$$

then Theorem 3 gives (24). If condition (22) is valid and $s=0$, then the conditions of Theorem 4 are satisfied and any solution bounded on $[0,+\infty)$ tends to an equilibrium as $t \rightarrow+\infty$.

Note that for $\sigma=4, r=687.5$, and $a=0.0052$ the analytical estimate of the Lyapunov dimension of the corresponding self-excited attractor is as follows

$$
\operatorname{dim}_{L} K<2.890997461 \ldots
$$

and the values of the local Lyapunov dimension at equilibria are

$$
\operatorname{dim}_{L} S_{0}=2.890833450 \ldots, \quad \operatorname{dim}_{L} S_{1,2}=2.009763700 \ldots
$$

Numerically, by an algorithm in Appendix D, the Lyapunov dimension of the selfexcited attractor is $\mathrm{LD}=2.1405$.

The analytical estimate of the Lyapunov dimension of the hidden attractor for $\sigma=4, r=700$, and $a=0.0052$ is as follows

$$
\operatorname{dim}_{L} K<2.891882349 \ldots,
$$

and the local Lyapunov dimension at the stationary points are the following

$$
\operatorname{dim}_{L} S_{0}=2.891767634 \ldots, \operatorname{dim}_{L} S_{1,2}=1.966483617 \ldots
$$

Numerically, the Lyapunov dimension of the hidden attractor is LD $=2.1322$.

Thus, the Lyapunov dimensions of B-attractor (which involve equilibrium $S_{0}$ ) and the global attractor are very close to the analytical estimate.

In the general case the coincidence of the analytical upper estimate with the local Lyapunov dimension at a stationary point gives the exact value of the Lyapunov dimension of the global attractor (see, e.g., studies of various Lorenz-like systems $[90,94,97,99,109,114])$. 


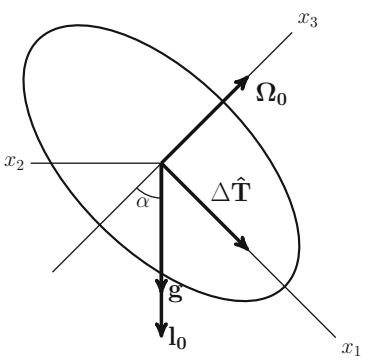

Fig. 11. Illustration of the problem.

\section{Appendix}

\section{A Description of the physical problem}

Consider the convection of viscous incompressible fluid motion inside the ellipsoid

$$
\left(\frac{x_{1}}{a_{1}}\right)^{2}+\left(\frac{x_{2}}{a_{2}}\right)^{2}+\left(\frac{x_{3}}{a_{3}}\right)^{2}=1, \quad a_{1}>a_{2}>a_{3}>0
$$

under the condition of stationary inhomogeneous external heating. We assume that the ellipsoid and heat sources rotate with constant velocity $\Omega_{0}$ around the axis. Vector $\mathbf{l}_{\mathbf{0}}$ determines the orientation of the ellipsoid and has the same direction as the gravity vector $\mathbf{g}$. Vector $\mathbf{g}$ is stationary with respect to the ellipsoid motion. The value $\Omega_{\mathbf{0}}$ is assumed to be such that the centrifugal forces can be neglected when compared with the influence of the gravitational field. Consider the case when the ellipsoid rotates around the axis $x_{3}$ that has a constant angle $\alpha$ with gravity vector $\mathbf{g}(|\mathbf{g}|=g)$. The vector $\mathbf{g}$ is placed in the plane $x_{1} x_{3}$. Then, $\Omega_{\mathbf{0}}=\left(0,0, \Omega_{0}\right)$ and $\mathbf{l}_{\mathbf{0}}=\left(a_{1} \sin \alpha, 0,-a_{3} \cos \alpha\right)$. Let the steady-state temperature difference $\Delta \hat{\mathbf{T}}=\left(q_{0}, 0,0\right)$ be generated along the axis $x_{1}$ (Fig. 11). The corresponding mathematical model (three-mode model of convection) was obtained by Glukhovsky and Dolzhansky [48] in the form (see (3))

$$
\left\{\begin{array}{c}
\dot{x}=-\sigma x+C z+A y z \\
\dot{y}=R_{a}-y-x z \\
\dot{z}=-z+x y
\end{array}\right.
$$

Here

$$
\begin{aligned}
\sigma & =\frac{\lambda}{\mu}, \quad T_{a}=\frac{\Omega_{0}^{2}}{\lambda^{2}}, \quad R_{a}=\frac{g \beta a_{3} q_{0}}{2 a_{1} a_{2} \lambda \mu}, \\
A & =\frac{a_{1}^{2}-a_{2}^{2}}{a_{1}^{2}+a_{2}^{2}} \cos ^{2} \alpha T_{a}^{-1}, \quad C=\frac{2 a_{1}^{2} a_{2}}{a_{3}\left(a_{1}^{2}+a_{2}^{2}\right)} \sigma \sin \alpha, \\
x(t) & =\mu^{-1}\left(\omega_{3}(t)+\frac{g \beta a_{3} \cos \alpha}{2 a_{1} a_{2} \Omega_{0}} q_{3}(t)\right), \quad y(t)=\frac{g \beta a_{3}}{2 a_{1} a_{2} \lambda \mu} q_{1}(t), \\
z(t) & =\frac{g \beta a_{3}}{2 a_{1} a_{2} \lambda \mu} q_{2}(t),
\end{aligned}
$$

and $\lambda, \mu, \beta$ are the coefficients of viscosity, heat conduction, and volume expansion, respectively; $q_{1}(t), q_{2}(t)$, and $q_{3}(t)\left(q_{3}(t) \equiv 0\right)$ are temperature differences on the 
principal axes of the ellipsoid; $\omega_{1}(t), \omega_{2}(t)$, and $\omega_{3}(t)$ are the projections of the vectors of fluid angular velocities on the axes $x_{1}, x_{2}$, and $x_{3}$, respectively. Here

$$
\omega_{1}(t)=-\frac{g \beta a_{3}}{2 a_{1} a_{2} \Omega_{0}} \cos \alpha q_{1}(t), \quad \omega_{2}(t)=-\frac{g \beta a_{3}}{2 a_{1} a_{2} \Omega_{0}} \cos \alpha q_{2}(t) .
$$

The parameters $\sigma, T_{a}$, and $R_{a}$ are the Prandtl, Taylor, and Rayleigh numbers, respectively.

The linear change of variables [48]

$$
x \rightarrow x, \quad y \rightarrow C^{-1} y, \quad z \rightarrow C^{-1} z,
$$

transforms system (3) into the system

$$
\left\{\begin{array}{c}
\dot{x}=-\sigma x+z+A_{c} y z \\
\dot{y}=R_{c}-y-x z \\
\dot{z}=-z+x y
\end{array}\right.
$$

with

$$
R_{c}=R_{a} C, \quad A_{c}=\frac{A}{C^{2}} .
$$

After the linear transformation (see, e.g., [99]):

$$
x \rightarrow x, \quad y \rightarrow R_{c}-\frac{\sigma}{R_{c} A_{c}+1} z, \quad z \rightarrow \frac{\sigma}{R_{c} A_{c}+1} y,
$$

system (25) takes the form of (1) with

$$
a=\frac{A_{c} \sigma^{2}}{\left(R_{c} A_{c}+1\right)^{2}}, \quad r=\frac{R_{c}}{\sigma}\left(R_{c} A_{c}+1\right) .
$$

\section{B Proof of Proposition 1}

For system (1) the characteristic polynomial of the Jacobian matrix of the right-hand side at the point $\mathrm{x}_{0}=\left(x_{0}, y_{0}, z_{0}\right) \in \mathbb{R}^{3}$ has the form

$$
\chi\left(\mathrm{x}_{0}\right)=\lambda^{3}+p_{1}\left(\mathrm{x}_{0}\right) \lambda^{2}+p_{2}\left(\mathrm{x}_{0}\right) \lambda+p_{3}\left(\mathrm{x}_{0}\right),
$$

where

$$
\begin{aligned}
& p_{1}\left(\mathrm{x}_{0}\right)=\sigma+2 \\
& p_{2}\left(\mathrm{x}_{0}\right)=x_{0}^{2}+a y_{0}^{2}-a z_{0}^{2}+(\sigma+a r) z_{0}-r \sigma+2 \sigma+1, \\
& p_{3}\left(\mathrm{x}_{0}\right)=\sigma x_{0}^{2}+a y_{0}^{2}-a z_{0}^{2}-2 a x_{0} y_{0} z_{0}+(\sigma+a r) x_{0} y_{0}+(\sigma+a r) z_{0}-r \sigma+\sigma .
\end{aligned}
$$

Applying the Hurwitz criterion, the necessary and sufficient stability conditions for stationary point $\mathrm{x}_{0}$ are the following

$$
\begin{gathered}
p_{1}\left(\mathrm{x}_{0}\right)>0 \\
p_{2}\left(\mathrm{x}_{0}\right)>0 \\
p_{3}\left(\mathrm{x}_{0}\right)>0, \text { and } \\
p_{1}\left(\mathrm{x}_{0}\right) p_{2}\left(\mathrm{x}_{0}\right)-p_{3}\left(\mathrm{x}_{0}\right)>0 .
\end{gathered}
$$


Equilibria $S_{1,2}$ exist for $r>1$, and we can check that $\chi\left(S_{1}\right)=\chi\left(S_{2}\right)$. For further analysis we can introduce

$$
D=a\left(\sqrt{(\sigma-a r)^{2}+4 \sigma a}-(\sigma-a r)\right)>0 .
$$

Then for stationary points (5), condition (29) takes the form

$$
p_{2}\left(S_{1,2}\right)=\frac{2}{D}\left(C_{1} \sqrt{(\sigma-a r)^{2}+4 \sigma a}-C_{1}(\sigma-a r)-2 \sigma^{2} a(\sigma-a r)\right)>0
$$

where

$$
C_{1}=\sigma(\sigma-a r)^{2}+a^{2} r+a \sigma^{2}>0 .
$$

Because $\sigma>a r>0$, we have

$$
\begin{aligned}
C_{1} \sqrt{(\sigma-a r)^{2}+4 \sigma a} & >C_{1}(\sigma-a r)+2 \sigma^{2} a(\sigma-a r) \quad \text { iff } \\
(\sigma-a r)^{2}+4 \sigma a & >\left((\sigma-a r)+\frac{2 \sigma^{2} a(\sigma-a r)}{C_{1}}\right)^{2} \text { iff } \\
4 \sigma a> & \frac{4 \sigma^{2} a(\sigma-a r)^{2}}{C_{1}}+\frac{4 \sigma^{4} a^{2}(\sigma-a r)^{2}}{C_{1}^{2}} \text { iff } \\
C_{1}^{2} & >\sigma(\sigma-a r)^{2} C_{1}+\sigma^{3} a(\sigma-a r)^{2} .
\end{aligned}
$$

The last inequality is satisfied because

$$
\frac{1}{a}\left(C_{1}^{2}-\sigma(\sigma-a r)^{2} C_{1}-\sigma^{3} a(\sigma-a r)^{2}\right)=\sigma a r(\sigma-a r)^{2}+a\left(\sigma^{2}+a r\right)^{2}>0 .
$$

This implies (33).

Condition (30) for $S_{1,2}$ takes the form

$$
\begin{aligned}
p_{3}\left(S_{1,2}\right)= & \frac{2 \sigma}{D}\left(\sqrt{(\sigma-a r)^{2}+4 \sigma a}-(\sigma-a r+2 a)\right) \\
& \left.\cdot\left((\sigma-a r)^{2}+4 \sigma a-(\sigma-a r) \sqrt{(\sigma-a r)^{2}+4 \sigma a}\right)\right)>0 .
\end{aligned}
$$

Since

$$
(\sigma-a r)^{2}+4 \sigma a-(\sigma-a r+2 a)^{2}=4 a^{2}(r-1)>0
$$

and

$$
(\sigma-a r)^{2}+4 \sigma a>(\sigma-a r) \sqrt{(\sigma-a r)^{2}+4 \sigma a} \quad \text { iff } \quad \sqrt{(\sigma-a r)^{2}+4 \sigma a}>(\sigma-a r),
$$

condition (34) is also satisfied.

Condition (31) for stationary points $S_{1,2}$ is as follows

$$
\begin{aligned}
& p_{1}\left(S_{1,2}\right) p_{2}\left(S_{1,2}\right)-p_{3}\left(S_{1,2}\right)=\frac{2}{D}\left(C_{2} \sqrt{(\sigma-a r)^{2}+4 \sigma a}-\right. \\
& \left.-C_{2}(\sigma-a r)-2 \sigma^{2} a(\sigma(\sigma-a r)-4 a)\right)>0
\end{aligned}
$$

where

$$
C_{2}=\left((\sigma(\sigma-a r)-a)^{2}+a^{2}(r \sigma+2 r-1)+a \sigma^{2}(\sigma-2)\right) .
$$


If $\sigma>2$, then $C_{2}>0$ and we can derive a chain of inequalities for (35):

$$
\begin{aligned}
C_{2} \sqrt{(\sigma-a r)^{2}+4 \sigma a} & >C_{2}(\sigma-a r)+2 \sigma^{2} a(\sigma(\sigma-a r)-4 a) \text { iff } \\
(\sigma-a r)^{2}+4 \sigma a & >\left((\sigma-a r)+\frac{2 \sigma^{2} a(\sigma(\sigma-a r)-4 a)}{C_{2}}\right)^{2} \text { iff } \\
4 \sigma a & >\frac{4 \sigma^{2} a(\sigma-a r)(\sigma(\sigma-a r)-4 a)}{C_{2}}+\frac{4 \sigma^{4} a^{2}(\sigma(\sigma-a r)-4 a)^{2}}{C_{2}^{2}} \text { iff } \\
C_{2}^{2} & >\sigma(\sigma-a r)(\sigma(\sigma-a r)-4 a) C_{2}+\sigma^{3} a(\sigma(\sigma-a r)-4 a)^{2} .
\end{aligned}
$$

We can divide the last inequality by $\left(-a^{2}\right)$ and rewrite it in the form of polynomial

$$
\begin{array}{r}
a^{2} \sigma^{2}(\sigma-2) r^{3}-a\left(2 \sigma^{4}-4 \sigma^{3}-3 a \sigma^{2}+4 a \sigma+4 a\right) r^{2}+\sigma^{2}\left(\sigma^{3}+2(3 a-1) \sigma^{2}-\right. \\
-8 a \sigma+8 a) r-\sigma^{3}\left(\sigma^{3}+4 \sigma^{2}-16 a\right)<0 .
\end{array}
$$

This inequality corresponds to the stability condition for the equilibria $S_{1,2}$.

\section{Proofs of Lemma 1 and the completeness of system (1)}

Suppose that the Lyapunov function has the form

$$
V(x, y, z)=\frac{1}{2}\left[x^{2}+y^{2}+(a+1)\left(z-\frac{\sigma+r}{a+1}\right)^{2}\right] .
$$

Here $V(x, y, z) \rightarrow \infty$ as $|(x, y, z)| \rightarrow \infty$. For an arbitrary solution $\mathrm{x}(t)=$ $(x(t), y(t), z(t))$ of system (1) we have

$$
\begin{aligned}
\dot{V}(x, y, z) & =x(-\sigma x+\sigma y-a y z)+y(r x-y-x z)+((a+1) z-(\sigma+r))(-z+x y) \\
& =-\sigma x^{2}-y^{2}-(a+1) z^{2}+(\sigma+r) z .
\end{aligned}
$$

Suppose that $\varepsilon \in(0,(a+1))$ and $c=\min \{\sigma, 1,(a+1)-\varepsilon\}>0$. Then

$$
\begin{aligned}
\dot{V}(x, y, z) & =-\sigma x^{2}-y^{2}-((a+1)-\varepsilon) z^{2}-\varepsilon z^{2}+(\sigma+r) z \\
& =-\sigma x^{2}-y^{2}-((a+1)-\varepsilon) z^{2}-\left(\sqrt{\varepsilon} z-\frac{(\sigma+r)}{2 \sqrt{\varepsilon}}\right)^{2}+\frac{(\sigma+r)^{2}}{4 \varepsilon} \\
& \leq-c\left(x^{2}+y^{2}+z^{2}\right)+\frac{(\sigma+r)^{2}}{4 \varepsilon} .
\end{aligned}
$$

Suppose that $x^{2}+y^{2}+z^{2} \geq R^{2}$. Then a positive $\varkappa$ exists such that

$$
\dot{V}(x, y, z) \leq-c R^{2}+\frac{(\sigma+r)^{2}}{4 \varepsilon}<-\varkappa \quad \text { for } \quad R^{2}>\frac{1}{c} \frac{(\sigma+r)^{2}}{4 \varepsilon} .
$$

We choose a number $\eta>0$ such that

$$
\{(x, y, z) \mid V(x, y, z) \leq \eta\} \supset\left\{(x, y, z) \mid x^{2}+y^{2}+z^{2} \leq R^{2}\right\},
$$

i.e., the relation $x^{2}+y^{2}+z^{2} \leq R^{2}$ implies that

$$
x^{2}+y^{2}+(a+1)\left(z-\frac{\sigma+r}{a+1}\right)^{2}=x^{2}+y^{2}+z^{2}+a z^{2}-2(\sigma+r) z+\frac{(\sigma+r)^{2}}{a+1} \leq 2 \eta .
$$


Since

$$
-2(\sigma+r) z \leq 2(\sigma+r)|z| \leq 2(\sigma+r) R,
$$

it is sufficient to choose $\eta>0$ such that

$$
(a+1) R^{2}+2(\sigma+r) R+\frac{(\sigma+r)^{2}}{a+1} \leq 2 \eta, \quad \text { i.e. } \quad \eta \geq \frac{1}{2}(a+1)\left(R+\frac{\sigma+r}{a+1}\right)^{2} .
$$

Further, we can apply Theorem 2, which implies the Lemma.

Using Lyapunov function (36), we can prove the boundedness of solutions of system (1) for $t \leq 0$. Note that

$$
\left(2\left(z-\frac{\sigma+r}{a+1}\right)^{2}+\frac{(\sigma+r)^{2}}{2(a+1)^{2}}\right)-\left(z-\frac{\sigma+r}{2(a+1)}\right)^{2}=\left(z-\frac{3(\sigma+r)}{2(a+1)}\right)^{2} \geq 0
$$

so the inequality

$$
\left(z-\frac{\sigma+r}{2(a+1)}\right)^{2} \leq\left(2\left(z-\frac{\sigma+r}{a+1}\right)^{2}+\frac{(\sigma+r)^{2}}{2(a+1)^{2}}\right)
$$

is satisfied. This implies that

$$
\begin{aligned}
\dot{V} & \geq 2 \sigma\left(-\frac{1}{2} x^{2}\right)+2\left(-\frac{1}{2} y^{2}\right)-2(a+1)\left[\left(z-\frac{\sigma+r}{a+1}\right)^{2}+\frac{(\sigma+r)^{2}}{4(a+1)^{2}}\right]+\frac{1}{4} \frac{(\sigma+r)^{2}}{a+1} \\
& =2 \sigma\left(-\frac{1}{2} x^{2}\right)+2\left(-\frac{1}{2} y^{2}\right)+4\left(-\frac{1}{2}(a+1)\left(z-\frac{\sigma+r}{a+1}\right)^{2}\right)-\frac{1}{4} \frac{(\sigma+r)^{2}}{a+1} \\
& \geq 2 \sigma(-V)+2(-V)+4(-V)-\frac{1}{4} \frac{(\sigma+r)^{2}}{a+1}
\end{aligned}
$$

Suppose that $k=2 \sigma+2+4$, and $m=\frac{1}{4} \frac{(\sigma+r)^{2}}{a+1}$. Then

$$
\dot{V}+k V \geq-m
$$

This implies that

$$
\frac{d}{d t}\left(e^{k t} V\right)=e^{k t} \dot{V}+k e^{k t} V \geq-e^{k t} m
$$

Thus for $t \leq 0$ we have

$$
V(0)-e^{k t} V(t) \geq\left(m e^{k t}-m\right) / k
$$


or

$$
V(t) \leq e^{-k t} V(0)+\left(m e^{-k t}-m\right) / k
$$

This implies that $V$ does not tend to infinity in a finite negative time. Therefore, any solution $(x(t), y(t), z(t))$ of system (1) does not tend to infinity in a finite negative time. Thus, differential Eq. (1) generates a dynamical system for $t \in \mathbb{R}$.

\section{Computation of Lyapunov exponents and Lyapunov dimension using MATLAB}

The singular value decomposition $(S V D)$ of a fundamental matrix $\mathrm{X}(t)$ has the from

$$
\mathrm{X}(t)=\mathrm{U}(t) \Sigma(t) \mathrm{V}^{T}(t): \quad \mathrm{U}(t)^{T} \mathrm{U}(t) \equiv I \equiv \mathrm{V}(t)^{T} \mathrm{~V}(t),
$$

where $\Sigma(t)=\operatorname{diag}\left\{\sigma_{1}(t), \ldots, \sigma_{n}(t)\right\}$ is a diagonal matrix with positive real diagonal entries known as singular values. The singular values are the square roots of the eigenvalues of the matrix $\mathrm{X}(t)^{*} \mathrm{X}(t)$ (see [54]). Lyapunov exponents are defined as the upper bounds of the exponential growth rate of the singular values of the fundamental matrix of linearized system (see Eq. (13)).

We now give a MATLAB implementation of the discrete SVD method for computing Lyapunov exponents based on the product SVD algorithm (see, e.g., [40, 159]).

Listing 1: productsSVD.m - product SVD algorithm.

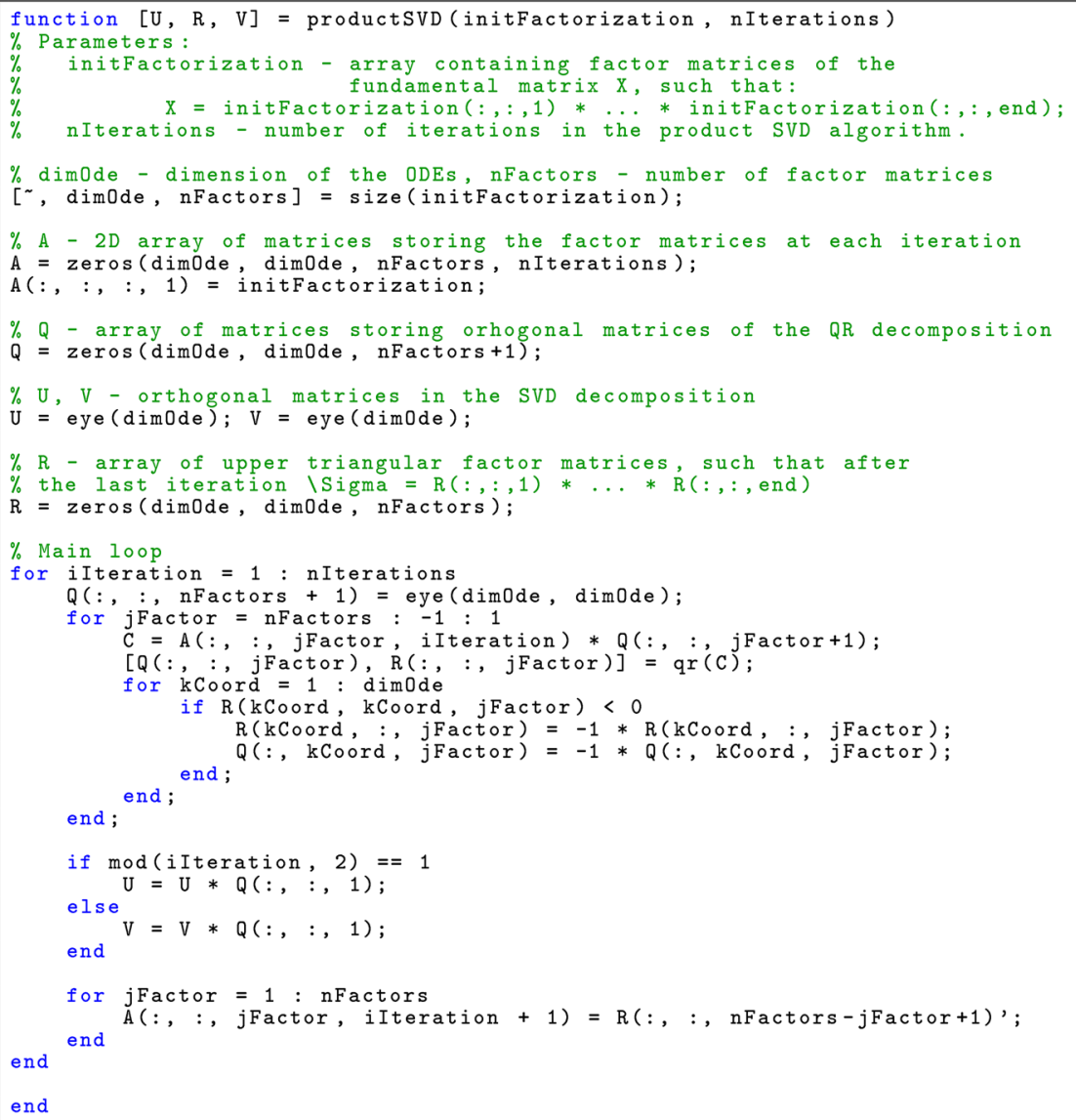


Listing 2: computeLEs.m - computation of the Lyapunov exponents.

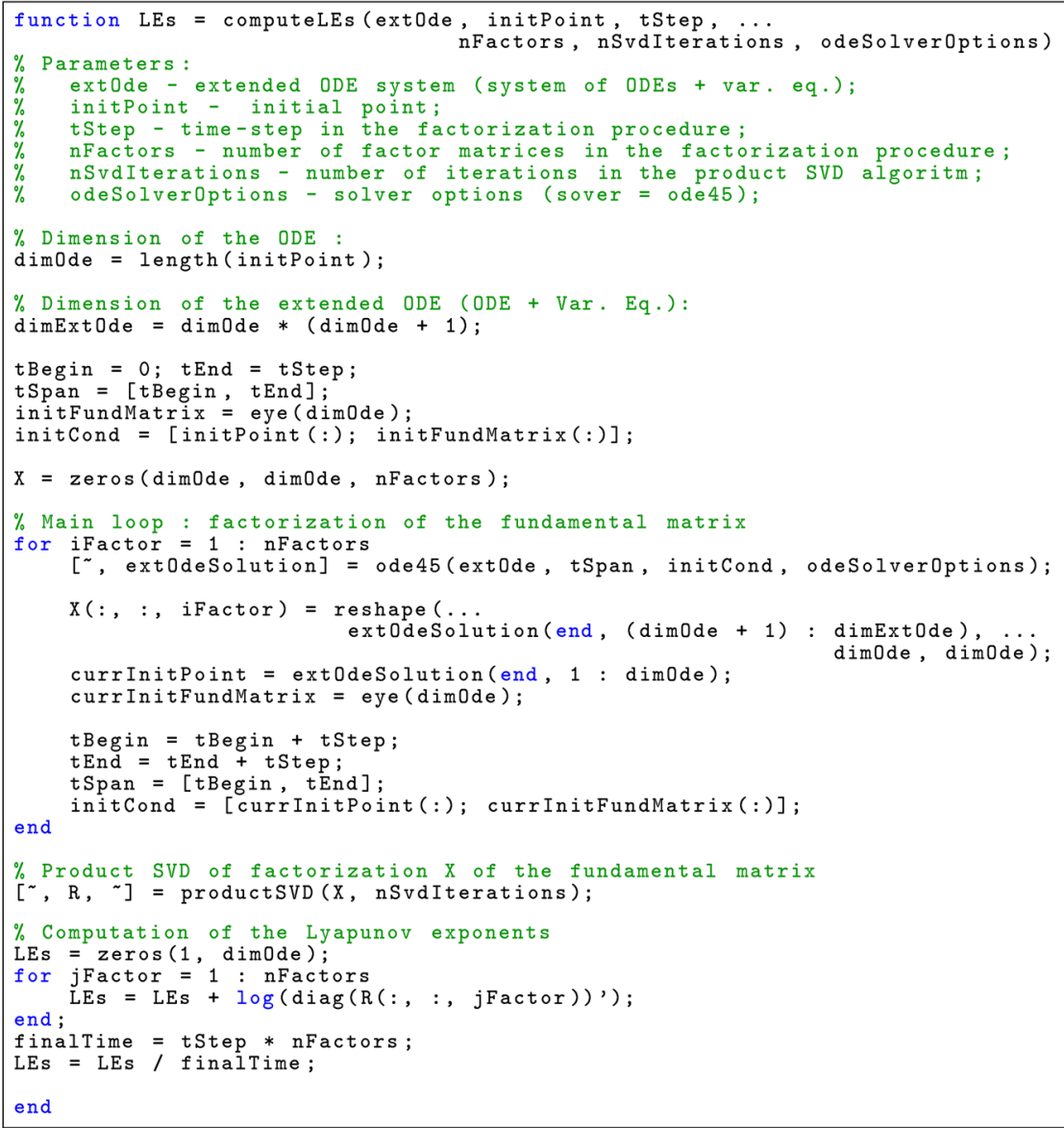

Listing 3: lyapunovDim.m - computation of the Lyapunov dimension.

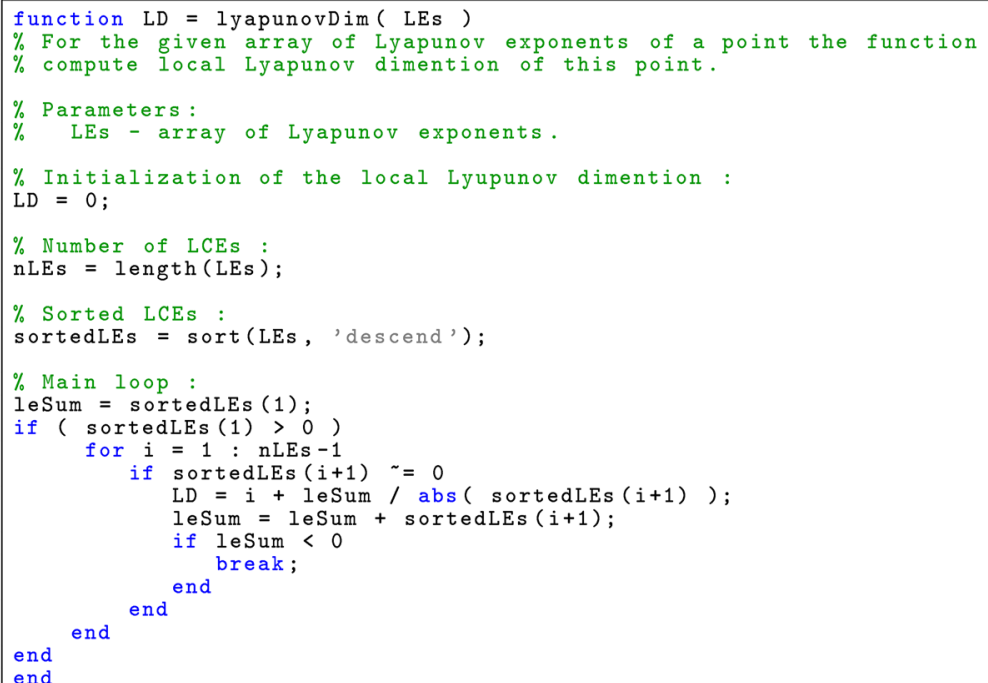


Listing 4: genLorenzSyst.m - generalized Lorenz system (1) along with the variational equation.

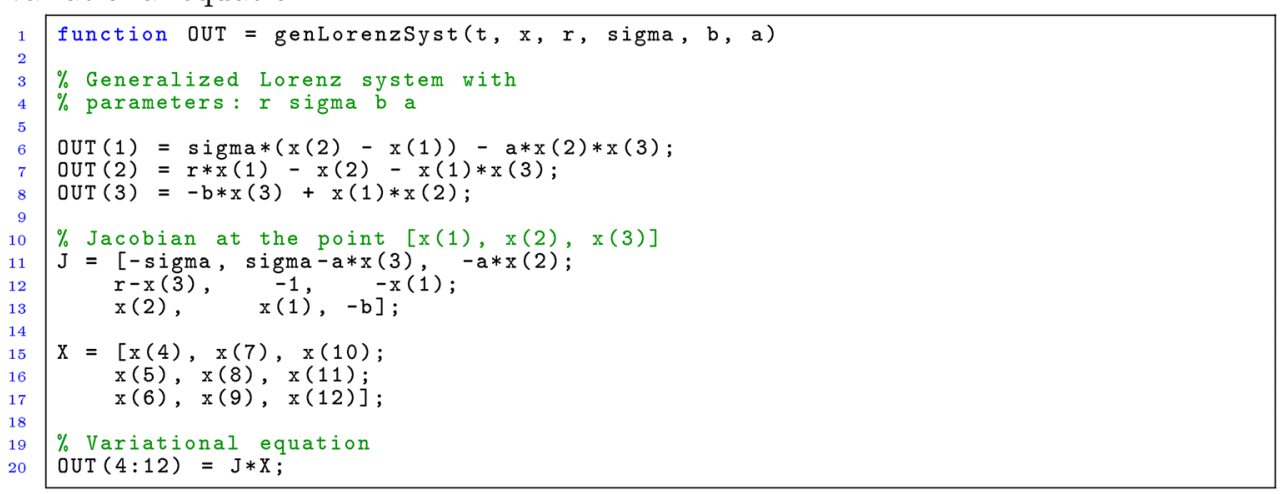

Listing 5: main.m - computation of the Lyapunov exponents and local Lyapunov dimension for the hidden attractor of generalized Lorenz system (1).

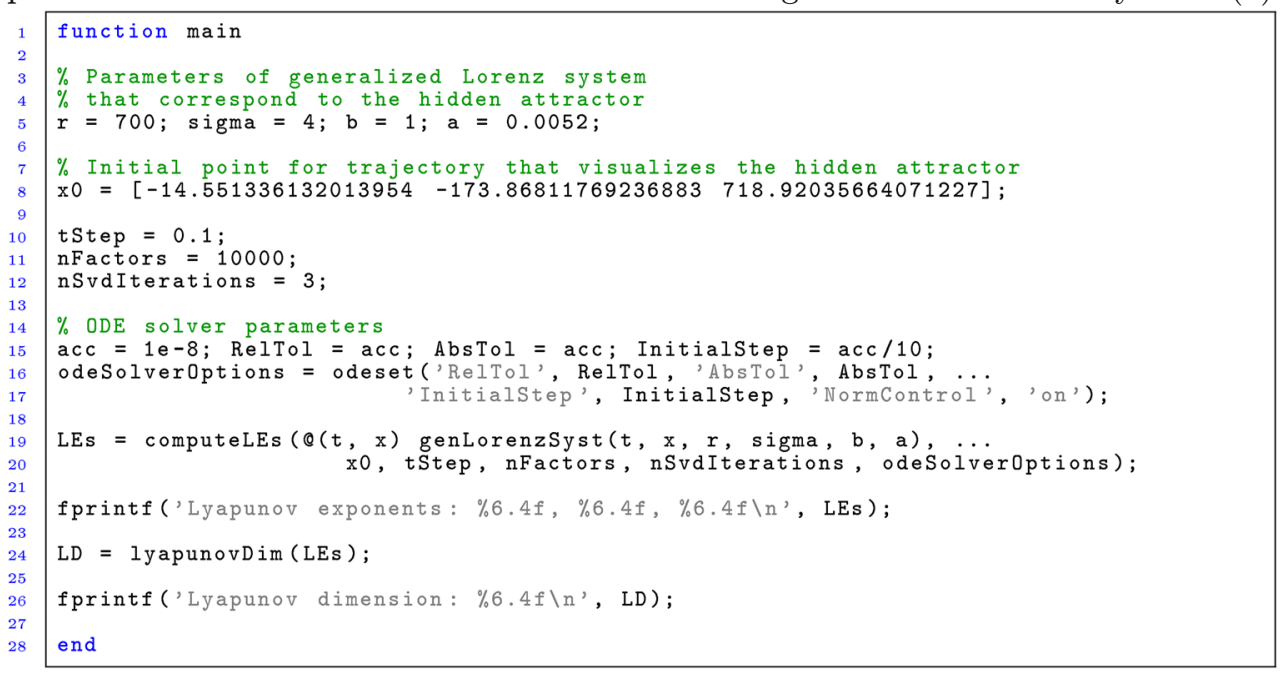

\section{E Fishing principle and the existence of a homoclinic orbit in the Glukhovsky-Dolghansky system}

\section{E.1 Fishing Principle}

Consider autonomous system of differential equations (1) with the parameter

$$
\dot{\mathrm{x}}=\mathrm{f}(\mathrm{x}, q), \quad t \in \mathbb{R}, \mathrm{x} \in \mathbb{R}^{n}, q \in \mathbb{R}^{m} .
$$

Let $\gamma(s), s \in[0,1]$ be a smooth path in the space of the parameter $\{q\}=\mathbb{R}^{m}$. Consider the following Tricomi problem [162]: Is there a point $q_{0} \in \gamma(s)$ for which system (37) with $q_{0}$ has a homoclinic trajectory?

Consider system (37) with $q=\gamma(s)$, and introduce the following notions. Let $\mathrm{x}(t, s)^{+}$be an outgoing separatrix of the saddle point $\mathrm{x}_{0}$ (i.e. $\lim _{t \rightarrow-\infty} \mathrm{x}(t, s)^{+}=\mathrm{x}_{0}$ ) with 


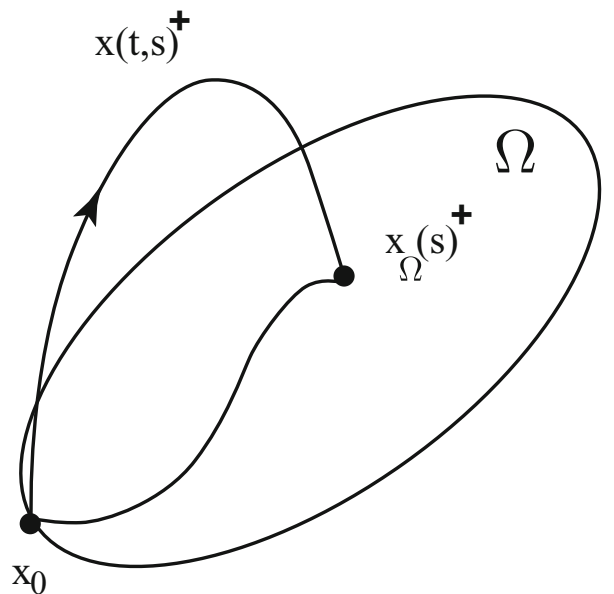

(a) Separatrix $\mathrm{x}(t, s)^{+}$, where $s \in$ $\left[0, s_{0}\right)$.

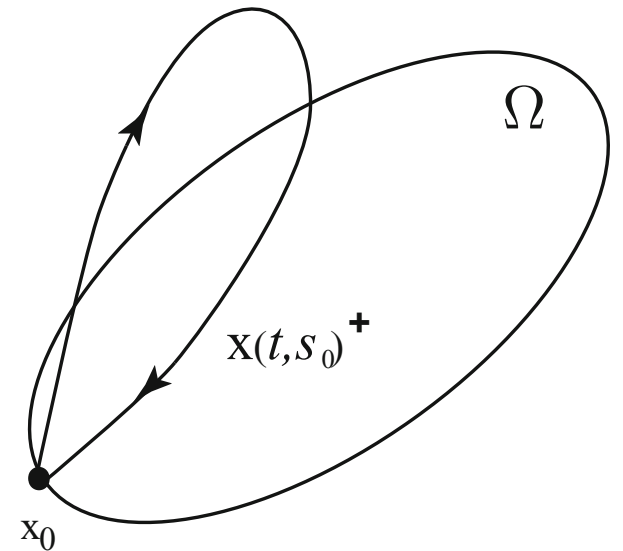

(b) Separatrix $\mathrm{x}(t, s)^{+}$, where $s=s_{0}$.

Fig. 12. Separatrix $\mathrm{x}(t, s)^{+}$, where $s \in\left[0, s_{0}\right]$.

a one-dimensional unstable manifold. Define by $\mathrm{x}_{\Omega}(s)^{+}$the point of the first crossing of separatrix $\mathrm{x}(t, s)^{+}$with the closed set $\Omega$ :

$$
\begin{gathered}
\mathrm{x}(t, s)^{+} \bar{\in} \Omega, \quad t \in(-\infty, T), \\
\mathrm{x}(T, s)^{+}=\mathrm{x}_{\Omega}(s)^{+} \in \Omega .
\end{gathered}
$$

If there is no such crossing, we assume that $\mathrm{x}_{\Omega}(s)^{+}=\emptyset$ (the empty set).

Theorem 6 (Fishing Principle $[\mathbf{8 7}, \mathbf{9 6}, \mathbf{9 8}])$. Suppose that for the path $\gamma(s)$ there is an $(n-1)$-dimensional bounded manifold $\Omega$ with a piecewise-smooth edge $\partial \Omega$ that possesses the following properties.

1. For any $\mathrm{x} \in \Omega \backslash \partial \Omega$ and $s \in[0,1]$, the vector $f(\mathrm{x}, \gamma(s))$ is transversal to the manifold $\Omega \backslash \partial \Omega$,

2. for any $s \in[0,1], f\left(\mathrm{x}_{0}, \gamma(s)\right)=0$, the point $\mathrm{x}_{0} \in \partial \Omega$ is a saddle;

3. for $s=0$ the inclusion $\mathrm{x}_{\Omega}(0)^{+} \in \Omega \backslash \partial \Omega$ is valid (Fig. 12a),

4. for $s=1$ the relation $\mathrm{x}_{\Omega}(1)^{+}=\emptyset$ is valid (i.e. $\mathrm{x}_{\Omega}(1)^{+}$is an empty set),

5. for any $s \in[0,1]$ and $\mathrm{y} \in \partial \Omega \backslash \mathrm{x}_{0}$ there exists a neighborhood $U(\mathrm{y}, \delta)=\{\mathrm{x}|| \mathrm{x}-$ $\mathrm{y} \mid<\delta\}$ such that $\mathrm{x}_{\Omega}(s)+\bar{\epsilon} U(\mathrm{y}, \delta)$.

If conditions 1)-5) are satisfied, then there exists $s_{0} \in[0,1]$ such that $\mathrm{x}\left(t, s_{0}\right)^{+}$is a homoclinic trajectory of the saddle point $\mathrm{x}_{0}$ (Fig. 12b).

The fishing principle can be interpreted as follows. Figure 12a shows a fisherman at the point $\mathrm{x}_{0}$ with a fishing $\operatorname{rod} \mathrm{x}(t, s)^{+}$. The manifold $\Omega$ is a lake surface and $\partial \Omega$ is a shore line. When $s=0$, a fish has been caught by the fishing rod. Then, $\mathrm{x}(t, s)^{+}, s \in\left[0, s_{0}\right)$ is a path taken by the fishing rod when it brings the fish to the shore. Assumption 5) implies that the fish cannot be taken to the shore $\partial \Omega \backslash \mathrm{x}_{0}$, because $\partial \Omega \backslash \mathrm{x}_{0}$ is a forbidden zone. Therefore, only the situation shown in Fig. 12b is possible (i.e., at $s=s_{0}$ the fisherman has caught a fish). This corresponds to a homoclinic orbit.

Now let us describe the numerical procedure for defining the point $\Gamma$ on the path $\gamma(s)$, which corresponds to a homoclinic trajectory. Here we assume that conditions 
1), 2), and 5) of the fishing Principle are satisfied. Consider a sequence of paths $\gamma_{j}(s) \subset\left\{\gamma_{j-1}(s), s \in[0,1]\right\} \subset\{\gamma(s), s \in[0,1]\}, \forall s \in[0,1]$ such that the length $\left\{\gamma_{j}(s)\right\}$ tends to zero as $j \rightarrow+\infty$. Condition 3$)$ is satisfied for $\gamma_{j}(0)$ and condition $4)$ is satisfied for $\gamma_{j}(1)$. This sequence can be obtained if the paths $\gamma$ and $\gamma_{j}$ are sequentially divided into two paths of the same length and we choose the path such that for its end points condition 3) is satisfied and condition 4) is not satisfied (or vice versa). Obviously, the sequence $\gamma_{j}(s), s \in[0,1]$ is contracted to the point $\Gamma \in$ $\left\{\gamma_{j}(s), s \in[0,1]\right\}, \forall j$. This point corresponds to a homoclinic trajectory of system (37).

Now, consider the conditions of the non-existence of a homoclinic orbit. Consider the Jacobian matrix of system (37)

$$
J(\mathrm{x}, s)=\frac{\partial \mathrm{f}}{\partial \mathrm{x}}(\mathrm{x}, \gamma(s))
$$

Let $\lambda_{1}(\mathrm{x}, s, S) \geqslant \cdots \geqslant \lambda_{n}(\mathrm{x}, s, S)$ denote the eigenvalues of the symmetrized matrix

$$
\frac{1}{2}\left(S J(\mathrm{x}, s) S^{-1}+\left(S J(\mathrm{x}, s) S^{-1}\right)^{*}\right)
$$

where $S$ is a nonsingular matrix.

Suppose system (37) has a saddle point $\mathrm{x}_{0} \equiv \mathrm{x}_{0}(s), \forall s \in[0,1]$, the point $\mathrm{x}_{0}$ belongs to a positively invariant bounded set $K$, and $J\left(\mathrm{x}_{0}, s\right)$ has only real eigenvalues.

Theorem 7 ([87]). Assume that there are a continuously differentiable scalar function $\vartheta(\mathrm{x}, s)$ and a nonsingular matrix $S$ such that for system $(37)$ with $q=\gamma(s)$, the inequality

$$
\lambda_{1}(\mathrm{x}, S)+\lambda_{2}(\mathrm{x}, S)+\dot{\vartheta}(\mathrm{x})<0, \forall \mathrm{x} \in K, \quad \forall s \in[0,1]
$$

is satisfied. Then system (37) has no homoclinic trajectories for all $s \in[0,1]$ such that

$$
\lim _{t \rightarrow-\infty} \mathrm{x}(t)=\lim _{t \rightarrow+\infty} \mathrm{x}(t)=\mathrm{x}_{0}
$$

\section{E.2 Existence of a homoclinic trajectories in the Glukhovsky-Dolzhansky system}

Consider the separatrix $x^{+}(t), y^{+}(t), z^{+}(t)$ of the zero saddle point of system (1), where $x(t)^{+}>0, \forall t \in(-\infty, \tau), \tau$ is a number, and $\lim _{t \rightarrow-\infty} x(t)^{+}=0$ (i.e. positive outgoing separatrix is considered).

Define the manifold $\Omega$ as

$$
\Omega=\left\{x=0, y \leq 0, y(\sigma-a z) \leq 0, y^{2}+z^{2} \leq 2 r^{2}\right\} .
$$

\section{Check condition 1 ).}

Inside the set $\Omega \backslash \partial \Omega$ we have

$$
\dot{x}=y(\sigma-a z)<0 .
$$

Check condition 5).

a) On $B_{1}=\{x=0, y=0,-\sqrt{2} r \leq z \leq \sigma / a\}$ system (1) has the solution

$$
x(t) \equiv y(t) \equiv 0, \quad z(t)=z(0) \exp (-t) .
$$

b) On $B_{2}=\left\{x=0, y<0, z=\sigma / a, y^{2}+z^{2} \leq 2 r^{2}\right\}$ we have

$$
\ddot{x}=\sigma y \text {. }
$$




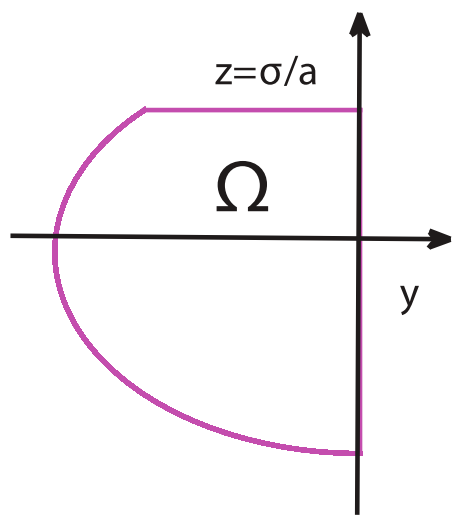

Fig. 13. Manifold $\Omega$.

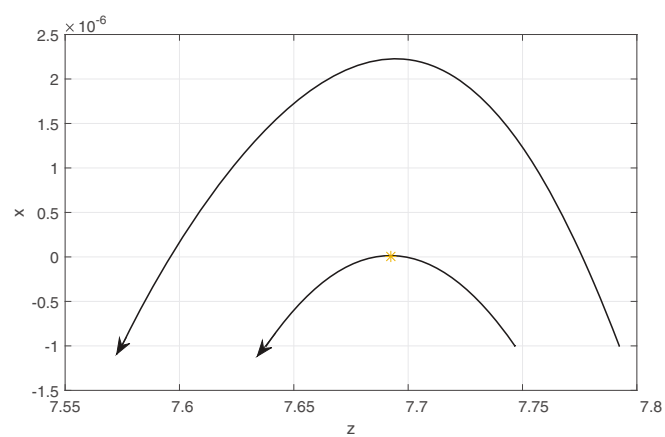

Fig. 14. Local behavior of the trajectories of system (1) in the neighborhood of set $B_{2}$ $\left(\sigma=4, a=0.52, r=10^{5}\right)$.

Therefore the local behavior of trajectories in the neighborhood of $B_{2}$ is shown in Fig. 14.

c) The set $B_{3}=\left\{x=0, y<0,-\sqrt{2} r \leq z \leq \sigma / a, y^{2}+z^{2}=2 r^{2}\right\}$ is located outside of the positively invariant cylinder $C$ (see Eq. (10)). Thus, the separatrices of the zero saddle point (which belongs to $C$ ) can not reach the set $B_{3}$.

Check condition 3).

Consider the development of the asymptotic integration of system (1) [88]. Assume that

$$
a r=c-\lambda \varepsilon+O\left(\varepsilon^{2}\right)
$$

where $c$ and $\lambda$ are some numbers and $\varepsilon=1 / \sqrt{r}$ is a small parameter.

Lemma 2. For any $\sigma>c, \sigma>1$ there exists a time $T>0$ such that for sufficiently large $r,\left(x^{+}(T), y^{+}(T), z^{+}(T)\right) \in \Omega \backslash \partial \Omega$ (i.e. condition 3) of the fishing principle is valid).

Proof (sketch). Using the transformation

$$
t \rightarrow \frac{t}{\sqrt{r}}, \quad x \rightarrow \sqrt{r} x, \quad y \rightarrow r y, \quad z \rightarrow r z,
$$


we can obtain

$$
\begin{aligned}
& \dot{x}=\sigma y-\varepsilon \sigma x-\left(c-\lambda \varepsilon+O\left(\varepsilon^{2}\right)\right) y z \\
& \dot{y}=x-\varepsilon y-x z \\
& \dot{z}=-\varepsilon z+x y .
\end{aligned}
$$

a) Consider the zero approximation of system (42) (system (42) without $\lambda \varepsilon$ and $\varepsilon=0)$ and its solution $\left(x_{0}(t), y_{0}(t), z_{0}(t)\right)$. There are two independent integrals

$$
\begin{aligned}
& V\left(x_{0}(t), y_{0}(t), z_{0}(t)\right)=(\sigma-c) z_{0}(t)^{2}+\sigma y_{0}(t)^{2}-x_{0}(t)^{2}=C_{1}, \\
& W=y_{0}(t)^{2}+z_{0}(t)^{2}-2 z_{0}(t)=C_{2} .
\end{aligned}
$$

Thus, the positive outgoing separatrix $x_{0}^{+}(t), y_{0}^{+}(t), z_{0}^{+}(t)$ of the saddle point $(x=y=$ $z=0$ ) of zero approximation of system (42) belongs to the intersection of surfaces $V=0$ and $W=0$, i.e.

$$
V\left(x_{0}^{+}(t), y_{0}^{+}(t), z_{0}^{+}(t)\right)=0=W\left(x_{0}^{+}(t), y_{0}^{+}(t), z_{0}^{+}(t)\right), \forall t \in(-\infty,+\infty) .
$$

From (43) it follows that $x_{0}^{+}(t) \neq 0, \forall t \in \mathbb{R}^{n}$ and

$$
\lim _{t \rightarrow+\infty} x_{0}^{+}(t)=\lim _{t \rightarrow+\infty} y_{0}^{+}(t)=\lim _{t \rightarrow+\infty} z_{0}^{+}(t)=0 .
$$

b) Consider the first approximation of system (42) (system (42) without $\left.O\left(\varepsilon^{2}\right)\right)$. For the small values of $\varepsilon$ the outgoing separatrix $\left(x_{1}^{+}(t), y_{1}^{+}(t), z_{1}^{+}(t)\right)$ of the zero saddle point of the first approximation of system (42) is close to $\left(x_{0}^{+}(t), y_{0}^{+}(t), z_{0}^{+}(t)\right)$ on $(-\infty, \tau)$. Therefore for sufficiently small $\varepsilon$ and some $\tau$ the separatrix $\left(x_{1}^{+}(t), y_{1}^{+}(t), z_{1}^{+}(t)\right)$ reaches $\delta$-vicinity of the zero saddle. Then there exists finite $\tau=\tau(\varepsilon, \delta)$ such that

$$
\left|x_{1}^{+}(\tau(\varepsilon, \delta))\right|<\delta, \quad\left|y_{1}^{+}(\tau(\varepsilon, \delta))\right|<\delta, \quad\left|z_{1}^{+}(\tau(\varepsilon, \delta))\right|<\delta .
$$

Consider two functions

$$
V_{\varepsilon}(x, y, z)=(\sigma-c+\lambda \varepsilon) z^{2}+\sigma y^{2}-x^{2}, \quad W=y^{2}+z^{2}-2 z .
$$

For the derivatives of (44) along the positive outgoing separatrix we have

$$
\begin{aligned}
\frac{d}{d t} V_{\varepsilon}(t) & \equiv \frac{d}{d t} V_{\varepsilon}\left(x_{1}^{+}(t), y_{1}^{+}(t), z_{1}^{+}(t)\right)=-2 \varepsilon V_{\varepsilon}\left(x_{1}^{+}(t), y_{1}^{+}(t), z_{1}^{+}(t)\right)+2 \varepsilon(\sigma-1) x_{1}^{+}(t)^{2} \\
\frac{d}{d t} W(t) & \equiv \frac{d}{d t} W\left(x_{1}^{+}(t), y_{1}^{+}(t), z_{1}^{+}(t)\right)=-2 \varepsilon W\left(x_{1}^{+}(t), y_{1}^{+}(t), z_{1}^{+}(t)\right)-2 \varepsilon z_{1}^{+}(t) .
\end{aligned}
$$

Integrating (45) from $-\infty$ to $\tau$ and taking into account

$$
\lim _{\tau_{0} \rightarrow-\infty} V_{\varepsilon}\left(\tau_{0}\right)=\lim _{\tau_{0} \rightarrow-\infty} W\left(\tau_{0}\right)=0
$$

we obtain

$$
V_{\varepsilon}(\tau)=2 \varepsilon(\sigma-1) \int_{-\infty}^{\tau} e^{-2 \varepsilon(\tau-s)}\left(x_{1}^{+}(t)\right)^{2} d t=2 \varepsilon(\sigma-1) M_{0}+o(\varepsilon)
$$




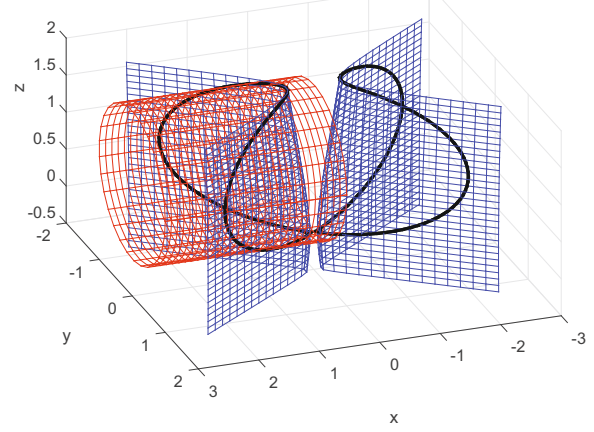

(a) Zero approximation of system (42): integrals ( $V$ - blue, $W-$ red) and separatrices (black)

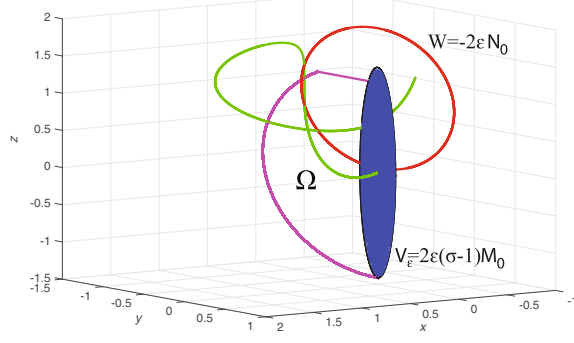

(b) First approximation of system (42): for $x_{1}^{+}(T)=0$ the positive outgoing separatrix is located inside magenta domain $\Omega$, and near red circuit and the border of blue ellipsoid (48)

Fig. 15. $\sigma=2.3445, a=0.0065, r=300, c=2, \lambda=1$.

$$
W(\tau)=-2 \varepsilon \int_{-\infty}^{\tau} e^{-2 \varepsilon(\tau-s)}\left(z_{1}^{+}(s)\right)^{2} d s=-2 \varepsilon N_{0}+o(\varepsilon),
$$

where $M_{0}$ and $N_{0}$ are some positive numbers. If $z_{1}$ and $z_{2}$ satisfy

$$
V_{\varepsilon}\left(0,0, z_{1}\right)=2 \varepsilon(\sigma-1) M_{0}, \quad W\left(0,0, z_{2}\right)=-2 \varepsilon N_{0},
$$

then

$$
z_{1}=\sqrt{\varepsilon} \sqrt{\frac{2 M_{0}(\sigma-1)}{\sigma-c+\lambda \varepsilon}}, \quad z_{2}=\varepsilon \frac{2 N_{0}}{1+\sqrt{1-2 N_{0} \varepsilon}} .
$$

Hence the situation shown in Fig. 15b occurs in the neighborhood of the saddle point $(x=y=z=0)$ for the surfaces

$$
V_{\varepsilon}(x, y, z)=2 \varepsilon(\sigma-1) M_{0}, \quad W(x, y, z)=2 \varepsilon N_{0} .
$$

The separatrix $\left(x_{1}^{+}(\tau), y_{1}^{+}(\tau), z_{1}^{+}(\tau)\right.$ has to be near the surfaces $V_{\varepsilon}(x, y, z)$ and $W(x, y, z)$. From the mutual disposition of surfaces (50) and different order of smallness in (49) it follows that if $x_{1}^{+}(\tau)>0$, then $y_{1}^{+}(\tau)<0$ and $\dot{x}_{1}^{+}(\tau)<0$ for sufficiently small $\varepsilon$. Moreover, $\dot{x}_{1}^{+}(t)<0$ for $t>\tau$ and $x_{1}^{+}(t) \leq 0$. This implies the existence of $T>\tau$ such that $x_{1}^{+}(T)=0$. We can obtain similar results for the case $x_{1}^{-}(\tau)<0$ (then $y_{1}^{-}(\tau)>0$ ). The behavior of separatrix $\left(x^{+}(\tau), y^{+}(\tau), z^{+}(\tau)\right.$ is consistent with the behavior of $\left(x_{1}^{+}(\tau), y_{1}^{+}(\tau), z_{1}^{+}(\tau)\right.$.

Check condition 4). We now check condition 4) for system (1) with parameters (27). For this system it was proved [19, pp. 276-277, 269-272] that if

$$
R_{c}<\frac{4(\sigma+1)}{1+\sqrt{1+8 A_{c}(\sigma+1)}}
$$

then condition (38) of Theorem 7 is satisfied for $S=I, V(x, s) \equiv 0$. 
Now we can show that if (51) holds, then condition 4) for system (1) is also satisfied. Consider the path

$$
\begin{aligned}
& R_{c}(s), \quad A_{c}(s), \quad \sigma(s) \equiv \sigma, \\
& A_{c}(0)=0, \quad A_{c}(1)=A_{c}, \\
& R_{c}(s) \in\left(\frac{2 \sigma}{1+\sqrt{1+4 A_{c}(s) \sigma}}, \frac{4(\sigma+1)}{1+\sqrt{1+8 A_{c}(s)(\sigma+1)}}\right), \\
& R_{c}(0)=\sigma(1+\delta),
\end{aligned}
$$

where $\delta$ is a small positive number.

For $s=0$ condition 4 ) is satisfied (see, e.g., [89,96]). If for some $s_{1} \in[0,1]$ condition 4 ) is not satisfied, then condition 3 ) is satisfied for $s_{1}$. In this case Theorem 6 implies that there exists $s_{2} \in\left[0, s_{1}\right]$ for which a homoclinic trajectory exists. But $R_{c}(s)$ is chosen in such a way that conditions of Theorem 7 are valid and hence the homoclinic trajectories do not exist. This contradiction proves the fulfillment of condition 4) of Theorem 6 for all $s \in[0,1]$. Condition 6$)$ is checked.

Check condition 2). From (52) it is obvious that condition 2 is satisfied.

Remark 6. For $c=\sigma$ Lemma 2 is not valid since a positive outgoing separatrix of the zero approximation of system (42) follows a heteroclitic orbit

$$
\lim _{t \rightarrow+\infty} x_{0}^{0}(t)=\lim _{t \rightarrow+\infty} y_{0}^{0}(t)=\lim _{t \rightarrow+\infty} z_{0}^{0}(t)=2 .
$$

In this case we may consider a sequence of systems close to (1). For example, instead of (27) we can consider $a=a\left(\beta_{k}\right)=\frac{A_{c} \sigma\left(\sigma-\beta_{k}\right)}{\left(R_{c} A_{c}+1\right)^{2}}$, where $\beta_{k}$ are a small positive numbers and $\lim _{k \rightarrow+\infty} \beta_{k}=0$, such that path (52) satisfies condition 4) of the fishing principle. Then, using Lemma 2 and the fishing principle, we get the sequences of $r_{k}^{h}$ and corresponding homoclinic orbits. Choosing a convergent subsequence from $r_{k}^{h}$ and using Arzela-Ascoli theorem, we can justify the existence of a homoclinic orbit in the initial system with $a(0)$.

Note also that since $a$ and $r$ are varying in the asymptotic integration, $\partial \Omega$ is also varying.

Finally we get the following

Theorem 8. For any fixed $A_{c}>0, \sigma>1$ there exists a number

$$
R_{c} \in\left(\frac{2 \sigma}{1+\sqrt{1+4 A_{c} \sigma}},+\infty\right)
$$

such that system (1) with parameters (27) has a homoclinic trajectory of the zero saddle point.

This work was supported by Russian Scientific Foundation (project 14-21-00041) and SaintPetersburg State University.

\section{References}

1. V.S. Afraimovich, S.V. Gonchenko, L.M. Lerman, A.L. Shilnikov, D.V. Turaev, Regular Chaotic Dyn. 19, 435 (2014)

2. M.A. Aizerman Uspekhi Mat. Nauk (in Russian) 4, 187 (1949) 
3. S.N. Akhtanov, Z.Z. Zhanabaev, M.A. Zaks, Phys. Lett. A 377, 1621 (2013)

4. R. Alli-Oke, J. Carrasco, W. Heath, A. Lanzon, A robust Kalman conjecture for firstorder plants, In: Proc. IEEE Control and Decision Conference (2012)

5. B.R. Andrievsky, N.V. Kuznetsov, G.A. Leonov, A.Y. Pogromsky, IFAC Proc. Vol. (IFAC-PapersOnline) 5, 75 (2013)

6. B.R. Andrievsky, N.V. Kuznetsov, G.A. Leonov, S.M. Seledzhi, IFAC Proc. Vol. (IFACPapersOnline) 19, 37 (2013)

7. B. Aulbach, Nonlinear Anal.: Theory, Meth. Applications 7, 1431 (1983)

8. A.V. Babin, Global attractors in PDE, In: Handbook of dynamical systems, Vol. 1B, (Elsevier Science, 2006), p. 983

9. A.V. Babin, M.I. Vishik, Attractors of Evolution Equations (North-Holland, Amsterdam, 1992)

10. N.E. Barabanov, Sib. Math. J. 29, 333 (1988)

11. L. Barreira, K. Gelfert, Ergodic Theory Dyn. Syst, 31, 641 (2011)

12. N.N. Bautin, Mat. Sb. (N.S.) (in Russian) 30, 181 (1952)

13. B.P. Belousov, Collection of short papers on radiation medicine for 1958, Chap. A periodic reaction and its mechanism, Moscow: Med. Publ. (in Russian) (1959)

14. V.N. Belykh, Qualitative methods of the theory of nonlinear oscillations in point systems (in Russian), (Gorki University Press, Gorki, 1980)

15. J. Bernat, J. Llibre, Dyn. Continuous, Discr. Impulsive Syst. 2, 337 (1996)

16. R. Best, N. Kuznetsov, O. Kuznetsova, G. Leonov, M. Yuldashev, R. Yuldashev, A short survey on nonlinear models of the classic Costas loop: rigorous derivation and limitations of the classic analysis, In: American Control Conference (ACC), IEEE (2015) (accepted)

17. G. Birkhoff, Dynamical Systems. Amer. Math. Soc. (1927)

18. I. Blekhman, D. Indeitsev, A. Fradkov, IFAC Proc. Vol. (IFAC-PapersOnline) 3, 126 (2007)

19. V.A. Boichenko, G.A. Leonov, V. Reitmann, Dimension theory for ordinary differential equations (Teubner, Stuttgart, 2005)

20. V.O. Bragin, V.I. Vagaitsev, N.V. Kuznetsov, G.A. Leonov, J. Comp. Syst. Sci. Int. 50, $511(2011)$

21. I. Burkin, N. Khien, Differ. Equ. 50, 1695 (2014)

22. M. Cartwright, E. Littlewood, London Math. Soc. 20, 180 (1976)

23. S. Celikovsky, A. Vanecek, Kybernetika 30, 403 (1994)

24. U. Chaudhuri, A. Prasad, Phys. Lett. A 378, 713 (2014)

25. D. Cheban, P. Kloeden, B. Schmalfuss, Nonlinear Dyn. Syst. Theory 2, 9 (2002)

26. G. Chen, T. Ueta, Int. J. Bifurcat. Chaos 9, 1465 (1999)

27. M. Chen, M. Li, Q. Yu, B. Bao, Q. Xu, J. Wang Nonlinear Dynamics (2015), doi: 10.1007/s11071-015-1983-7

28. M. Chen, J. Yu, B.C. Bao, Electronics Lett. 51, 462 (2015)

29. V. Chepyzhov, A.Y. Goritskii, Adv. Sov. Math 10, 85 (1992)

30. V.V. Chepyzhov, M.I. Vishik, Attractors for equations of mathematical physics, Vol. 49, American Mathematical Society Providence, RI (2002)

31. L.O. Chua, M. Komuro, T. Matsumoto, IEEE Trans. Circ. Syst. CAS-33, 1072 (1986)

32. I. Chueshov, Introduction to the Theory of Infinite-dimensional Dissipative Systems, Electronic library of mathematics, ACTA (2002)

33. I. Chueshov, S. Siegmund, J. Dyn. Differential Equ. 17, 621 (2005)

34. I.D. Chueshov, Russian Math. Surveys 48, 135 (1993)

35. E.A. Coddington, Levinson, Theory of ordinary differential equations (Tata McGrawHill Education, 1995)

36. B. Coomes, J. Differential Equ. 82, 386 (1989)

37. P. Cvitanović, R. Artuso, R. Mainieri, G. Tanner, G. Vattay, Chaos: Classical and Quantum. Niels Bohr Institute, Copenhagen (2012), http://ChaosBook.org

38. X.Y. Dang, C.B. Li, B.C. Bao, H.G. Wu, Chin. Phys. B 24 (2015), Art. num. 050503 
39. M. Dellnitz, O. Junge, Set oriented numerical methods for dynamical systems, In: Handbook of dynamical systems, Vol. 2, (Elsevier Science, 2002), p. 221

40. L. Dieci, C. Elia, Math. Comput. Simul. 79, 1235 (2008)

41. G. Duffing, Erzwungene Schwingungen bei Veranderlicher Eigenfrequenz, F. Vieweg u. Sohn, Braunschweig (1918)

42. M. Eckert, Arnold Sommerfeld: Science, Life and Turbulent Times (Springer, 2013), p. 1868

43. Eckmann, J.P., Ruelle, D. Rev. Mod. Phys. 57, 617 (1985)

44. S. Evtimov, S. Panchev, T. Spassova, Comptes rendus de l'Académie bulgare des Sciences 53, 33 (2000)

45. R.E. Fitts, Trans. IEEE AC-11, 553 (1966)

46. P. Giesl, Construction of global Lyapunov functions using radial basis functions (Springer 2007)

47. H. Gingold, D. Solomon, Theory, Meth. Applications 74, 4234 (2011)

48. A.B. Glukhovskii, F.V. Dolzhanskii, Academy of Sciences, USSR, Izvestiya, Atmosph. Oceanic Phys. 16, 311 (1980)

49. P. Hartman, Ordinary Differential Equation (John Wiley, New York, 1964)

50. M. Henon, Commun. Math. Phys. 50, 69 (1976)

51. D. Hilbert, Bull. Amer. Math. Soc. (8), 437 (1901-1902)

52. M.W. Hirsch, S. Smale, R.L. Devaney, Pure and Applied Mathematics, Vol. 60 (Elsevier Academic Press, 2004)

53. W. Hoover, Phys. Rev. A 31, 1695 (1985)

54. R. Horn, C. Johnson, Topics in Matrix Analysis (Cambridge University Press, 1994)

55. B. Hunt, Nonlinearity 9, 845 (1996)

56. Y. Ilyashenko, L. Weign, Nonlocal Bifurcations American Mathematical Society (Providence, Rhode Island, 1999)

57. S. Jafari, J. Sprott, Chaos, Solitons Fractals 57, 79 (2013)

58. S. Jafari, J.C. Sprott, V.T. Pham, S.M.R.H. Golpayegani, A.H. Jafari, Int. J. Bifurcat. Chaos 24 (2014), Art. num. 1450134

59. R.E. Kalman, Trans. ASME 79, 553 (1957)

60. T. Kapitaniak, Phys. Rev. E 47, 1408 (1993)

61. T. Kapitaniak, Phys. Rev. E 53, 6555 (1996)

62. J.L. Kaplan, J.A. Yorke, Chaotic behavior of multidimensional difference equations, Functional Differential Equations and Approximations of Fixed Points (Springer, Berlin, 1979), p. 204

63. S. Kingni, S. Jafari, H. Simo, P. Woafo, Euro. Phys. J. Plus 129 (2014)

64. M.A. Kiseleva, N.V. Kuznetsov, Vestnik St. Petersburg Univ. Math. 48, 66 (2015)

65. M.A. Kiseleva, N.V. Kuznetsov, G.A. Leonov, P. Neittaanmäki, Drilling systems failures and hidden oscillations. In: IEEE 4th International Conference on Nonlinear Science and Complexity, NSC 2012 - Proceedings (2012), p. 109

66. P. Kloeden, M. Rasmussen, Nonautonomous Dynamical Systems (American Mathematical Society, 2011)

67. A. Kuznetsov, S. Kuznetsov, E. Mosekilde, N. Stankevich, J. Phys. A: Math. Theor. 48, 125 (2015)

68. N. Kuznetsov, O. Kuznetsova, G. Leonov, P. Neittaanmaki, M. Yuldashev, R. Yuldashev, Limitations of the classical phase-locked loop analysis, International Symposium on Circuits and Systems (ISCAS), IEEE (2015) (accepted)

69. N. Kuznetsov, O. Kuznetsova, G. Leonov, V. Vagaitsev, Informatics in Control, Automation and Robotics, Lecture Notes in Electrical Engineering, Vol. 174, Part 4, chap. Analytical-numerical localization of hidden attractor in electrical Chua's circuit (Springer, 2013), p. 149

70. N. Kuznetsov, G. Leonov, IFAC World Cong. 19, 5445 (2014)

71. N. Kuznetsov, G. Leonov, M. Yuldashev, R. Yuldashev, IFAC Proc. Vol. (IFACPapersOnline) 19, $8253(2014)$

72. N. Kuznetsov, G.A. Leonov, T.N. Mokaev, Hidden attractor in the Rabinovich system [arXiv: 1504.04723v1] (2015) 
73. N. Kuznetsov, V. Vagaitsev, G. Leonov, S. Seledzhi, Localization of hidden attractors in smooth Chua's systems, International Conference on Applied and Computational Mathematics (2011), p. 26

74. N.V. Kuznetsov, T. Alexeeva, G.A. Leonov, Invariance of Lyapunov characteristic exponents, Lyapunov exponents, and Lyapunov dimension for regular and non-regular linearizations [arXiv:1410.2016v2] (2014)

75. N.V. Kuznetsov, O.A. Kuznetsova, G.A. Leonov, Diff. Eq. Dyn. Syst. 21, 29 (2013)

76. N.V. Kuznetsov, O.A. Kuznetsova, G.A. Leonov, V.I. Vagaytsev, Automat. Robot. 1, $279(2011)$

77. N.V. Kuznetsov, G.A. Leonov, Izv. RAEN, Diff. Uravn. 5, 71 (2001)

78. N.V. Kuznetsov, G.A. Leonov, On stability by the first approximation for discrete systems, 2005 International Conference on Physics and Control, PhysCon 2005, Proceedings Vol. 2005 (IEEE, 2005), p. 596

79. N.V. Kuznetsov, G.A. Leonov, S.M. Seledzhi, Proc. Vol. (IFAC-PapersOnline) 18, 2506 (2011)

80. N.V. Kuznetsov, G.A. Leonov, V.I. Vagaitsev, IFAC Proc. Vol. (IFAC-PapersOnline) 4, $29(2010)$

81. N.V. Kuznetsov, T.N. Mokaev, P.A. Vasilyev, Commun. Nonlinear Sci. Numer. Simul. 19, 1027 (2014)

82. O. Ladyzhenskaya, Russian Math. Surv. 42, 25 (1987)

83. O.A. Ladyzhenskaya, Attractors for semi-groups and evolution equations (Cambridge University Press, 1991)

84. S.K. Lao, Y. Shekofteh, S. Jafari, J. Sprott, Int. J. Bifurcat. Chaos 24 (2014), Art. num. 1450010

85. F. Ledrappier, Comm. Math. Phys. 81, 229 (1981)

86. G. Leonov, St. Petersburg Math. J. 13, 453 (2002)

87. G. Leonov, Nonlinear Dyn. 78, 2751 (2014)

88. G. Leonov, Doklady Math. 462, 1 (2015)

89. G. Leonov, Phys. Lett. A 379, 524 (2015)

90. G. Leonov, N. Kuznetsov, N. Korzhemanova, D. Kusakin, Estimation of Lyapunov dimension for the Chen and Lu systems [arXiv:1504.04726v1] (2015)

91. G. Leonov, N. Kuznetsov, T. Mokaev, Homoclinic orbit and hidden attractor in the Lorenz-like system describing the fluid convection motion in the rotating cavity, Commun. Nonlinear Sci. Numer. Simul., doi:10.1016/j.cnsns.2015.04.007 (2015)

92. G. Leonov, V. Reitman, Attraktoreingrenzung fur nichtlineare Systeme (Teubner, Leipzig, 1987)

93. G.A. Leonov, J. Appl. Math. Mechan. 47, 861 (1983)

94. G.A. Leonov, Vestnik St. Petersburg Univ. Math. 24, 41 (1991)

95. G.A. Leonov, St. Petersburg Univ. Press, St. Petersburg (2008)

96. G.A. Leonov, Phys. Lett. A 376, 3045 (2012)

97. G.A. Leonov, J. Appl. Math. Mechan. 76(2), 129 (2012)

98. G.A. Leonov, Shilnikov chaos in Lorenz-like systems. Int. J. Bifurcat. Chaos 23 (2013), Art. num. 1350058

99. G.A. Leonov, V.A. Boichenko, Acta Applicandae Math. 26, 1 (1992)

100. G.A. Leonov, A.I. Bunin, N. Koksch, ZAMM - J. Appl. Math. and Mechanics / Z. Ang. Math. Mechanik 67, 649 (1987)

101. G.A. Leonov, I.M. Burkin, A.I., Shepelyavy, Frequency Methods in Oscillation Theory (Kluwer, Dordretch, 1996)

102. G.A. Leonov, N.V. Kuznetsov, Int. J. Bifurcat. Chaos 17, 1079 (2007)

103. G.A. Leonov, N.V. Kuznetsov, Localization of hidden oscillations in dynamical systems (plenary lecture), In: 4th International Scientific Conference on Physics and Control (2009). http://www.math.spbu.ru/user/leonov/publications/2009-PhysCon-Leonov-plenary-hidden-oscillations.pdf

104. G.A. Leonov, N.V. Kuznetsov, Doklady Math. 84, 475 (2011)

105. G.A. Leonov, N.V. Kuznetsov IFAC Proc. Vol. (IFAC-PapersOnline) 18, 2494 (2011) 
106. G.A. Leonov, N.V. Kuznetsov, IWCFTA2012 Keynote speech I - Hidden attractors in dynamical systems: From hidden oscillation in Hilbert-Kolmogorov, Aizerman and Kalman problems to hidden chaotic attractor in Chua circuits, In: IEEE 2012 Fifth International Workshop on Chaos-Fractals Theories and Applications (IWCFTA) (2012), p. XV

107. G.A. Leonov, N.V. Kuznetsov, Advances in Intelligent Systems and Computing, Vol. 210 AISC, Chap. Prediction of hidden oscillations existence in nonlinear dynamical systems: analytics and simulation (Springer, 2013), p. 5

108. G.A. Leonov, N.V. Kuznetsov, J. Bifurcat. Chaos 23 (2013)

109. G.A. Leonov, N.V. Kuznetsov, App. Math. Comp. 256, 334 (2015)

110. G.A. Leonov, N.V. Kuznetsov, M.A. Kiseleva, E.P. Solovyeva, A.M. Zaretskiy, Nonlinear Dyn. 77, 277 (2014)

111. G.A. Leonov, N.V. Kuznetsov, V.I. Vagaitsev, Phys. Lett. A 375, 2230 (2011)

112. G.A. Leonov, N.V. Kuznetsov, V.I. Vagaitsev, Physica D: Nonlinear Phenomena 241, $1482(2012)$

113. G.A. Leonov, O.A. Kuznetsova, Regul. Chaotic Dyn. 15, 354 (2010)

114. G.A. Leonov, A.Y. Pogromsky, K.E. Starkov, Phys. Lett. A 375, 1179 (2011)

115. G.A. Leonov, V. Reitmann, Math. Nachri. 129, 31 (1986)

116. N. Levinson, Ann. Math., 723 (1944)

117. N. Levinson, Ann. Math. 50, 127 (1949)

118. C. Li, J. Sprott, Phys. Lett. A 378, 178 (2014)

119. C. Li, J.C. Sprott, Coexisting hidden attractors in a 4-D simplified Lorenz system, Int. J. Bifurcat. Chaos 24 (2014) Art. num. 1450034

120. Q. Li, H. Zeng, X.S. Yang, Nonlinear Dyn. 77, 255 (2014)

121. B. Liao, Y.Y. Tang, L. An, Int. J. Wavelets, Multiresol. Inf. Proc. 8, 293 (2010)

122. E.N. Lorenz, J. Atmos. Sci. 20, 130 (1963)

123. J. Lu, G. Chen, Int. J. Bifurcat. Chaos 12, 1789 (2002)

124. A.M. Lyapunov, The General Problem of the Stability of Motion, Kharkov (1892)

125. L. Markus, H. Yamabe, Osaka Math. J. 12, 305 (1960)

126. G.H. Meisters, Polynomial flows on $\mathbb{R}^{n}$, In: Proceedings of the Semester on Dynamical Systems, Autumn 1986, at the Stefan Banach International Mathematics Center. ul. Mokotowska, 25 (Warszawa, Poland, 1989)

127. V. Melnikov, Trans. Moscow Math. Soc. 12, 1 (1963)

128. J. Milnor, Attractor. Scholarpedia 1 (2006)

129. M. Molaie, S. Jafari, J. Sprott, S. Golpayegani Int. J. Bifurcat. Chaos 23 (2013), Art. num. 1350188

130. S. Nose, Molecular Phys. 52, 255 (1984)

131. V. Oseledec, Multiplicative ergodic theorem: Characteristic Lyapunov exponents of dynamical systems, Transactions of the Moscow Mathematical Society 19, 179 (1968)

132. G. Osipenko, Banach Center Publ. 47, 173 (1999)

133. S. Panchev, T. Spassova, N.K. Vitanov, Chaos, Solitons Fractals 33, 1658 (2007)

134. V.T. Pham, S. Jafari, C. Volos, X. Wang, S. Golpayegani, Int. J. Bifurcat. Chaos 24, (2014)

135. V.T. Pham, F. Rahma, M. Frasca, L. Fortuna, Int. J. Bifurcat. Chaos 24 (2014)

136. V.T. Pham, C. Volos, S. Jafari, X. Wang, Optoelectronics Adv. Mater. Rapid Comm. 8, $535(2014)$

137. V.T. Pham, C. Volos, S. Jafari, X. Wang, S. Vaidyanathan, Optoelectronics Adv. Mater. Rapid Comm. 8, 1157 (2014)

138. V.T. Pham, C. Volos, S. Jafari, Z. Wei, X. Wang, Int. J. Bifurcat. Chaos 24, 1450073 (2014)

139. V.T. Pham, C. Volos, S. Vaidyanathan, T. Le, V. Vu, J. Eng. Sci. Tech. Rev. 2, 205 (2015)

140. A.S. Pikovski, M.I. Rabinovich, V.Y. Trakhtengerts, Sov. Phys. JETP 47, 715 (1978)

141. S. Pilyugin, Differential Equ. 47, 1929 (2011)

142. A. Pisarchik, U. Feudel, Phys. Reports 540, 167 (2014) 
143. V.A. Pliss, Some Problems in the Theory of the Stability of Motion. Izd LGU, Leningrad (in Russian) (1958)

144. B. van der Pol, Philosophical Mag. J. Sci. 7, 978 (1926)

145. A. Prasad, Int. J. Bifurcat. Chaos 25 (2015)

146. M. Rabinovich, Uspehi Physicheskih Nauk [in Russian] 125, 123 (1978)

147. V. Rasvan, Three lectures on dissipativeness, In: Automation, Quality and Testing, Robotics, 2006 IEEE International Conference on, Vol. 1 (IEEE, 2006), p. 167

148. J.W.S. Rayleigh, The theory of sound (Macmillan, London 1877)

149. V. Reitmann, Dynamical systems, attractors and there dimension estimates (Saint Petersburg State University Press, Saint Petersburg, 2013)

150. O.E. Rossler, Phys. Lett. A 57, 397 (1976)

151. G.R. Sell, J. Dyn. Differential Equ. 8, 1 (1996)

152. P. Sharma, M. Shrimali, A. Prasad, N.V. Kuznetsov, G.A. Leonov, Int. J. Bifurcat. Chaos 25 (2015)

153. L. Shilnikov, Sov. Math. Dokl. 6, 163 (1965)

154. A. Sommerfeld, Z. Vereins Deutscher Ingenieure 46, 391 (1902)

155. C. Sparrow, The Lorenz Equations: Bifurcations, Chaos, and Strange Attractors, Applied Mathematical Sciences (Springer, New York, 1982)

156. J. Sprott, Phys. Rev. E 50, R647 (1994)

157. J. Sprott, W. Hoover, C. Hoover, Phys. Rev. E 89 (2014)

158. J. Sprott, X. Wang, G. Chen, Int. J. Bifurcat. Chaos 23 (2013)

159. D.E. Stewart, Electr. Trans. Numer. Anal. 5, 29 (1997)

160. R. Temam, Infinite-dimensional Dynamical Systems in Mechanics and Physics, 2nd edn. (Springer-Verlag, New York, 1997)

161. G. Teschl, Graduate Studies in Mathematics, Vol. 140 (American Mathematical Soc., 2012)

162. F. Tricomi, Annali della R. Shcuola Normale Superiore di Pisa 2, 1 (1933)

163. Y. Ueda, N. Akamatsu, C. Hayashi, Trans. IEICE Japan 56A, 218 (1973)

164. A. Vannelli, M. Vidyasagar, Automatica 21, 69 (1985)

165. V. Venkatasubramanian, Stable operation of a simple power system with no equilibrium points, In: Proceedings of the 40th IEEE Conference on Decision and Control, Vol. 3 (2001), p. 2201

166. X. Wang, G. Chen, Nonlinear Dyn. 71, 429 (2013)

167. Z. Wei, I. Moroz, A. Liu, Turkish J. Math. 38, 672 (2014)

168. Z. Wei, R. Wang, A. Liu, Math. Comp. Simul. 100, 13 (2014)

169. Z. Wei, W. Zhang, Int. J. Bifurcat. Chaos 24 (2014)

170. Z. Wei, W. Zhang, Z. Wang, M. Yao, Int. J. Bifurcat. Chaos 25, 1550 (2015)

171. V.A. Yakubovich, Avtomation Remore Cont. 25, 905 (1964)

172. V.A. Yakubovich, G.A. Leonov, A.K. Gelig, Stability of Stationary Sets in Control Systems with Discontinuous Nonlinearities (World Scientific, Singapure, 2004)

173. T. Yoshizawa, Stability theory by Liapunov's second method, Math. Soc. Japan (1966)

174. P. Yu, G. Chen, Int. J. Bifurcat. Chaos 14, 1683 (2004)

175. H. Zhao, Y. Lin, Y. Dai, Int. J. Bifurcat. Chaos 24 (2014)

176. Z. Zhusubaliyev, E. Mosekilde, Math. Comp. Simul. 109, 32 (2015)

177. V.I. Zubov, L.F. Boron, Methods of A.M. Lyapunov and their application (Noordhoff Groningen, 1964) 